\title{
On the warm bias along the South-West African Coast in coupled models: An oceanic perspective
}

\author{
by \\ Jinbo Wang \\ B.S. Meteorology, Lanzhou University, China \\ M.S. Atmospheric sciences, Peking University, China \\ Submitted in partial fulfillment of the requirements for the degree of \\ Master of Science \\ at the \\ MASSACHUSETTS INSTITUTE OF TECHNOLOGY \\ and the \\ WOODS HOLE OCEANOGRAPHIC INSTITUTION
}

September 2008

(C) Jinbo Wang, 2008. All rights reserved.

The author hereby grants to MIT and WHOI permission to reproduce and distribute publicly paper and electronic copies of this thesis document in whole or in part.

Author

MIT/WHOI joint program in Physical Oceanography

September, 2008

Certified by

Paola Malanotte-Rizzoli
Professor
Thesis Supervisor

Accepted by

Raffaele Ferrari

Associate Professor

Chairman, Joint Committee for Physical Oceanography 


\title{
On the warm bias along the South-West African Coast in coupled models: An oceanic perspective
}

\author{
by \\ Jinbo Wang \\ Submitted to the MIT/WHOI Joint Program in Physical Oceanography \\ on Septemeber 2008, in partial fulfillment of the requirements for \\ the degree of Master of Science
}

\begin{abstract}
Coupled ocean/atmosphere simulations exhibit systematic warm biases over the South West African (SWA) coastal region. Recent investigations indicate that coastal ocean dynamics may play an important role in determining the SST patterns, but none of them provide a detailed analysis. In this study, I analyze simulations produced both by coupled models and by idealized models. Then results are interpreted on the basis of a theoretical framework. Finally the conclusion is reached that the insufficient resolution of the ocean component in the coupled model is responsible for the warm biases over the SWA coastal region. The coarse resolution used in the ocean model has an artificially stretched coastal side-wall boundary layer, which induces a smaller upwelling velocity in the boundary layer. The vertical heat transport decreases even when the volume transport is unchanged because of its nonlinear relationship with the magnitude of the upwelling velocity. Based on the scaling of the idealized model simulations, a simplified calculation shows that the vertical heat transport is inversely proportional to the zonal resolution over the coastal region. Therefore, increasing the horizontal resolution can considerably improve the coastal SST simulation, and better resolve the coastal dynamics.
\end{abstract}

Thesis Supervisor: Paola Malanotte-Rizzoli

Title: Professor 


\section{Acknowledgments}

This study is a result of helps from many people. Hyodae Seo provided me coupled model outputs used in this study and clearified my confusion on coupled models. Jean-Michel Campin and Gael Forget helped me on running MITgcm. Conversations with Markus Jochum, Glenn Flierl, Steven Lentz, and Michael Spall were always thought-provoking, and their suggestions greatly helped me improve this thesis. Andrew Barton, Martha Buckley, Kjetil Våge helped me on the manuscript. I would like to thank them all. I am especially grateful to my advisor, Paola Malanotte-Rizzoli, for all the support, guidance and friendship, without which this work could not be accomplished.

I would like thank my friends in PAOC and MIT/WHOI Joint Program who helped make my time here delightful. Special thanks go to Christie Wood, Peter Sugimura, Evgeny Logvinov, together with whom I had a lot of enjoyable and memorable time.

Many thanks go to Ruixin Huang, who shared his knowledge, wisdom, and experience with me. My wife, Xiaoyan, was always beside me sharing every enjoyable and stressful moment in our life.

Thanks to all who helped me! 


\section{Contents}

1 Introduction 6

2 Model setup 12

3 Coupled simulations $\quad 18$

3.1 Sea surface temperature . . . . . . . . . . . . . . 18

3.2 Possible mechanisms . . . . . . . . . . . . . . . . 21

$3.2 \mathrm{a} \quad$ Net surface heat flux .................. 21

3.2b Alongshore wind stress . . . . . . . . . . . . . 22

3.2c Coastal oceanic processes .................. 24

3.3 Uncertainties . . . . . . . . . . . . . . . . 30

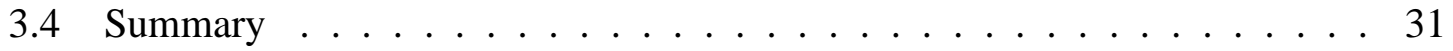

4 A two dimensional coastal upwelling model 33

4.1 Vertical velocity and Ekman pumping . . . . . . . . . . 36

4.2 Boundary layer during spin-up . . . . . . . . . . . . . . 37

4.3 Diffusive boundary layers . . . . . . . . . . . . . . . . . . . 39 
5.1 Results with resolved boundary layers . . . . . . . . . . . . . . 43

5.2 Results with unresolved boundary layers . . . . . . . . . . . . . 46

5.3 The effect of horizontal resolution on the heat transport equation. . . . . . . 51

5.4 Simple relationship between the zonal resolution and the surface cooling . . 51

5.5 Model parameterization for the coastal boundary layer . . . . . . . . . 57

6 Summary and conclusions $\quad 60$ 


\section{Chapter 1}

\section{Introduction}

The sea surface temperature (SST) over the upwelling region along the Southwest African (SWA) coast is poorly represented in most of the coupled climate models, yet is of great importance in determining the tropical Atlantic variability (TAV). Davey et al. (2002) showed that none of the fully-coupled climate models is capable of reproducing the correct zonal SST gradient over the equatorial Atlantic. They have a systematic warm bias in the SWA coastal region. More recent coupled model simulations indicate that even state-of-the-art coupled models suffer from the same problem (Large and Danabasoglu 2006). The sensitivity of Atlantic climate to SST over the SWA coastal upwelling region and the poor forecasting capability of coupled climate models suggest the necessity to study on why the coastal SST is poorly simulated in the coupled models.

For a long time, it has been recognized as a common bias that the sea surface temperature (SST) is too warm off the west coasts of South America and southern Africa. Mechoso et al. (1995) examined 11 coupled models focusing on the tropical Pacific region and concluded that they shared troublesome systematic errors. The anomalously warm SST in a broad region west of Peru in a band near $10^{\circ} \mathrm{S}$ is one of them. They proposed that stratus cloud effects and coastal effects may be responsible for the warm SSTs. In their study, all the models overestimated the incident shortwave radiation into the ocean in that region 
because of the deficiency of the stratus clouds simulation. The positive feedback between SST and stratus clouds enlarges the warm bias. Underestimated coastal upwelling may also contribute to the warm bias. The weak upwelling is likely related to underestimated surface winds along the Peruvian coast because the abrupt change from the Andes mountains to the ocean surface is not well resolved in spectral atmosphere models. Mechoso et al. (1995) stated that "the reason for these errors is unclear". Ten years after that study, the warm bias along coasts of South America and southern Africa in the coupled climate model is still a puzzle. Large and Danabasoglu (2006) discusse the upper ocean biases in the state-ofthe-art Community Climate System Model version 3 (CCSM3) simulations, and showed that the warm SST biases still occur off coasts of SWA, Peru-Ecuador-Chile (PEC), and Baja-Southern California (BSC). Very large biases extend thousands of kilometers along the coast, but only about $300 \mathrm{~km}$ offshore. The sensitivity of these biases to the oceanic resolution (Yeager et al. 2006) suggests that the ocean is at least partially responsible for them, even though quantitative analysis of possible mechanisms are still lacking. The warm biases remain significant in the simulation where the surface forcing is completely prescribed (Large and Danabasoglu 2006).

The correct simulation of the coastal SST must reproduce the meridional SST gradient and asymmetry about the equator, which affects the Intertropical Convergence Zone (ITCZ) (Xie and Philander 1994). Specifically in the Atlantic ocean sector, the impact of the SWA coastal region on the meridional migration of the ITCZ will further affect the TAV.

The SST simulation over the subtropical upwelling region is crucial for the ITCZ simulation. There are two distinctive modes associated with the year-to-year variation in the annual migration of the Atlantic marine ITCZ: the zonal mode and the meridional mode. The zonal mode can be explained by the Bjerknes feedback (Bjerknes 1966), which is used to explain the Pacific El Nino and Southern Oscillation (ENSO) mode. Using an atmospheric general circulation model (AGCM), a recent study confirms that during boreal summer the atmosphere responds to the equatorial Atlantic SST anomaly through the Bjerknes mechanism (Chang et al. 2000). The overall signal in the equatorial Atlantic, however, is weaker 
than that in the equatorial Pacific.

The meridional mode involves off-equatorial SST changes which are intimately linked to the surface heat fluxes, particularly to the latent heat flux (Carton et al. 1996). The meridional mode has been explained by the Wind-Evaporation-SST (WES) feedback mechanism (Xie and Philander 1994), a mechanism that was also first applied to the Pacific Ocean and supported by the observational evidence (Chiang et al. 2002). According to the WES mechanism, the meridional atmospheric pressure gradient will change in response to variations of the hemispheric SST gradient through the hydrostatic adjustment of the atmospheric boundary layer (Lindzen and Nigam 1987). Even a small SST anomaly over the subtropical upwelling region can change the hemispheric SST gradient in a significant manner. The meridional pressure gradient drives a cross-equator flow within the atmospheric surface boundary layer and then change the meridional position of the ITCZ. The Coriolis force deflects the flow to increase (decrease) the wind speed over the colder (warmer) regions, and further to induce a positive feedback enhancing the existing SST anomalies (Xie and Philander 1994). The SST variation over the SWA region modulates the meridional mode of the Atlantic ITCZ.

The SSTs over subtropical upwelling regions do not merely response to atmospheric changes passively. Oceanic processes must be considered. The latent heat flux induced by the surface wind has been considered to be the dominant factor responsible for the SST variability over off-equatorial regions (Carton et al. 1996) with the exception of subtropical upwelling areas along eastern oceanic boundaries. Studies by Alexander et al. (2000) and Chang et al. (2003) showed that the formation of the meridional mode during the boreal spring is mostly due to remote atmospheric influences and the regional thermodynamic feedback, and can be captured by coupling the atmosphere to a simple one-dimensional mixed layer ocean model. One exception, however, is that over the coastal area in the vicinity of the SWA coastal region, the strength of the SST variability is underestimated, which means that the ocean also plays an important role in determining the SST variability. It is, however, unclear how ocean dynamics affect the SST variability. In the Southern Atlantic Ocean, 
especially over the SWA coastal region, the situation is even worse because of the lack of observational data. It is unknown which factor is more important in affecting SST variations over the SWA coastal region, the atmospheric surface heat uptake or the oceanic upwelling.

It has been proposed that the ocean circulation could modulate the TAV by two mechanisms associated with the ocean dynamics and the concept of subtropical cells (STCs). STCs are shallow meridional overturning circulations that transport the water subducted in the eastern subtropical Atlantic Ocean in winter to the tropics and the equator, where the water upwells to the surface. The upwelled water is modified by air-sea fluxes and advected back to the subtropics by the poleward Ekman transport in both hemispheres. While the STCs have been observed in the Pacific Ocean (Johnson and McPhaden 1999; Johnson and Marshall 2002), they are not well documented in the Atlantic Ocean. The first mechanism, the so-called $\bar{v} T^{\prime}$ mechanism, originally proposed for the decadal modulation of the Pacific ENSO by Gu and Philander (1997), involves equatorward advection by the mean flow of the STCs of temperature anomalies formed in the subtropical subduction zones. The second one, the so-called $v^{\prime} \bar{T}$ mechanism, proposed by Kleeman et al. (1999) involves strength changes of the STCs that lead to varying amounts of cold water transported to the tropics by the surface Ekman flow. In the tropical Atlantic, the southern STC is much stronger than the northern one as a result of the interaction between the meridional over turning circulation (MOC) and the STCs (Jochum and Malanotte-Rizzoli 2001).

Figure(1-1) schematically shows the mean tropical Atlantic (TA) circulation and the mean STCs, in which one of the most unknown components is the upwelling regions along the African coast. The surface Ekman transports, directed poleward in both hemispheres, have a zonally integrated, annual mean divergence between $10^{\circ} \mathrm{S}$ and $10^{\circ} \mathrm{N}$ of $26 \mathrm{~Sv}$ from the National Centers for Environmental Prediction (NCEP) reanalysis wind stresses. The time series of the Ekman transports across $10^{\circ} \mathrm{N}$ and $10^{\circ} \mathrm{S}$ for the period of 1990-1999 from the NCEP and the ERS-1/2 scatterometer stresses, as well as their divergence, show variations of about $2 \mathrm{~Sv}$ amplitude at interannual time scales. These variations should be in large 
part produced by corresponding variations in the upwelling regions along the North and the South African coast.

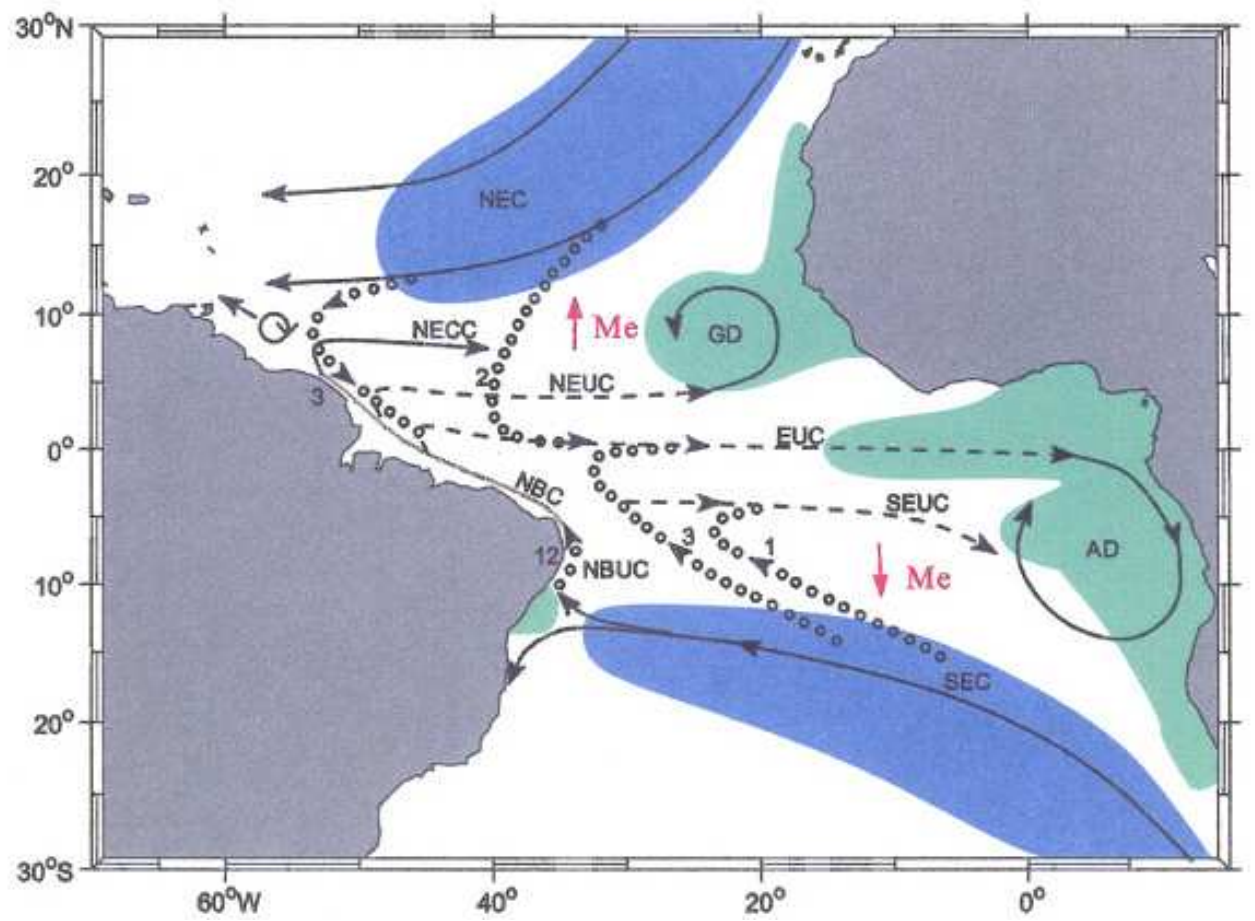

Figure 1-1: Schematic representation of the Tropical Atlantic circulation with subduction (blue) and upwelling (green) zones and Ekman transports in red. AD is the upwelling Angola Dome, also called the South West Africa (SWA) upwelling region, and GD the Guinea Dome. The subsurface equatorward thermocline pathways are dotted, both in the interior and along the western boundary. (TACE White Paper, 2003)

Although the upwelling region variations appear important for the TAV, the mechanisms are unclear. The warm biases in the climate model are related to the double ITCZ problem, through which TAV simulation is further affected. It is known that the resolving mesoscale features can affect the mean circulation and SST distributions. Danabasoglu et al. (2006) suggested that the warm SST biases observed along the eastern oceanic boundaries in most coupled models, in particular along the SWA coast, may be produced by the underestimated coastal upwelling. Seo et al. (2006) showed that increasing the horizontal resolution of the ocean model can partially eliminate the systematic bias over the SWA region. Jochum et al. (2005) argued that increasing the horizontal resolution in an ocean model removes the spu- 
rious horizontal diffusion of heat from the warm-pool to the Equatorial Counter-Current. This mechanism has been extended to the coastal upwelling region in the study of Seo et al. (2006), but detailed analysis of how the horizontal resolution affects coastal waters is still missing. In this study, I address the question of why there are warm biases over the SWA coastal region in coupled models from an oceanic perspective. It is proposed that the underestimated vertical heat transport along the boundary induced by misrepresented ocean dynamics within the eastern boundary layer produces the warm biases. This hypothesis is supported by three forced idealized ocean model simulations, where I show that the SST over the coastal upwelling region depends largely on how well the coastal boundary layer is resolved.

The paper is organized as follows. In Chapter 2, I describe the models and experimental setup. The major results from the coupled model are presented in Chapter 3, and the theory is reviewed in Chapter 4. The results from the idealized model are discussed in Chapter 5. Finally, other concluding remarks are given in Chapter 6. 


\section{Chapter 2}

\section{Model setup}

The importance of the oceanic effect on the SST simulation in the SWA coastal region can be tested by varying the horizontal resolution in the ocean model because higher horizontal resolution leads to better-resolved ocean dynamics. Four experiments are designed with different horizontal resolution in the ocean model, and they are 1) a coupled model with $1^{o}$ longitude $\times 1^{o}$ latitude horizontal resolution in the ocean model, 2) the same coupled model but with $\left.0.25^{\circ} \times 0.25^{\circ}, 3\right)$ an idealized ocean model with $1^{\circ} \times 1^{o}$ resolution, 4) the same idealized ocean model but with $0.25^{\circ} \times 0.25^{\circ}$. The coupled model has a real bathymetry and coastlines, and the idealized model has a square domain and a flat bottom. Comparisons of results from different experiments illustrate influence of the ocean dynamics on the SST simulation. Two more idealized experiments, which resolve the coastal boundary layer, are used as a theoretical guide to examine the effect of the varying horizontal resolution.

The coupled model is the Scripps Coupled Ocean Atmosphere Regional (SCOAR) model (Seo et al. 2007). It couples the atmospheric Regional Spectral Model(RSM) (Juang and Kanamitsu 1994; Juang et al. 1997) and the Regional Ocean Modeling System (ROMS) (Shchepetkin and McWilliams 2005). ROMS solves the incompressible, hydrostatic primitive equations with a free surface on horizontal curvilinear coordinates, and uses stretched general sigma coordinates to increase the vertical resolution near the surface and the bot- 
tom. The idealized experiments use the MITgcm (Marshall et al. 1997). It solves the incompressible hydrostatic/nonhydrostatic primitive equations on depth levels. The hydrostatic version is used in this study.

In the coupled model simulations, there are 30 vertical sigma layers, with roughly 10 layers in the upper 100 meters in the open ocean. The domain expands from $30^{\circ} \mathrm{N}$ to $30^{\circ} \mathrm{S}$ and from $90^{\circ} \mathrm{W}$ to $20^{\circ} \mathrm{E}$ covering the equatorial Atlantic. Mixed layer dynamics are parameterized using a KPP scheme (Large et al. 1994). A flux coupler was implemented by Seo et al. (2006) employing linear interpolation of surface fluxes (momentum, heat and moisture flux) from RSM to ROMS, and SST field from ROMS to RSM. For further technical details about SCOAR see Seo et al. (2006).

Two simulations were carried out by Seo et al. (2006) for the coupled model. In the first experiment both the atmospheric and oceanic models have the same low horizontal resolution, $1^{o} \times 1^{o}$. This experiment will be referred to as CL. In the second one, the resolution of the atmosphere RSM remains the same but the resolution of the ocean ROMS is increased to $0.25^{\circ} \times 0.25^{\circ}$. This experiment will be referred to as $\mathrm{CH}$. As the two experiments are otherwise identical, the difference between them evidences the effects of the better-resolved ocean on the TA circulation. In the work of Seo et al. (2006), the ocean model, ROMS, was first spun-up for 8 years as forced by the atmospheric climatology of COADS (da Silva et al. 1994). Initial and boundary conditions were from the World Ocean Atlas 2001 (Conkright et al. 2002). The final state of this run is used as the initial condition for the coupled simulations. The atmospheric field from the NCEP reanalysis (Kanamitsu et al. 2002) for the period 1998-2004 was then used as the forcing. CH and CL are both spun-up in the coupled mode for the year of 1998 to allow for surface adjustment processes. The years 1999 to 2004 are then used for the analysis.

The same configurations were also used to develop an idealized ocean-only model. I use the MITgcm with a $0.25^{\circ} \times 0.25^{\circ}$ horizontal resolution (referred as $\mathrm{IH}$ ), and a $1^{\circ} \times 1^{\circ}$ horizontal resolution (referred as IL). The idealized model simulations use a square domain with a grid setup similar to those used in the coupled model but with a flat bottom, cov- 
ering 60 degrees zonally and 80 degrees meridionally from $40^{\circ} \mathrm{S}$ to $40^{\circ} \mathrm{N}$. The eastern and western boundaries are walls, and the northern and southern boundaries are $5^{\circ}$-wide sponge layers with 40/4 days inner/outer relaxation time. This setup is further extended by raising the zonal resolution so that there is finer resolution of $0.05^{\circ}$ within a $2^{\circ}$-wide coastal region, and a coarser resolution in the interior, which linearly decreases from $0.05^{\circ}$ at the eastern boundary to $2.5^{\circ}$ at the western boundary. The meridional resolution is uniformly 1 degree. The resolution of $0.05^{\circ}$, about $5 \mathrm{~km}$, is sufficient to resolve the coastal sidewall boundary in the general circulation model considering that the first baroclinic Rossby radius of deformation is about $20 \mathrm{~km}$ to $40 \mathrm{~km}$ in the SWA coastal region. These simulations will be referred to $\mathrm{IHH}_{1}$, and $\mathrm{IHH}_{2}$, whose resolutions are identical but the parameterization coefficients are different as shown in Table 2.1. The different coefficients will alter the coastal side-wall boundary layer width which will be discussed in Chapter 4.

\section{Parameters in different simulations}

\begin{tabular}{|c|c|c|c|c|c|c|}
\hline \hline & $K_{H}$ & $A_{H}$ & $K_{V}$ & $A_{V}$ & $\sigma_{H}$ & $\sigma_{V}$ \\
\hline $\mathrm{IL}$ & 3000 & 5000 & $10^{-4}$ & $10^{-3}$ & $5 / 3$ & 10 \\
$\mathrm{IH}$ & 300 & 700 & $10^{-4}$ & $10^{-3}$ & $7 / 3$ & 10 \\
$I H H_{1}$ & 1000 & 2000 & $10^{-4}$ & $10^{-3}$ & 2 & 10 \\
$I H H_{2}$ & 1000 & 5000 & $10^{-4}$ & $10^{-3}$ & 5 & 10 \\
\hline
\end{tabular}

Table 2.1: Values of parameters for different simulations. $K_{H} / A_{H}$ are the horizontal diffusivity/viscosity coefficients, $K_{V} / A_{V}$ are the vertical diffusivity/viscosity coefficients, $\sigma_{H} / \sigma_{V}$ are horizontal/vertical Prandtl number which are discussed in Chapter 4

In the idealized experiments, the initial condition is a state of rest with a constant salinity of $35 \mathrm{psu}$ in space and time, and idealized zonally uniform temperature shown in figure (2-1). It is constructed as:

$$
T(z)_{(\lambda, \theta)}=T_{e q}(z) \times\left(28^{\circ} C-\frac{|\theta|}{\triangle \theta} \times 16^{\circ} C\right)
$$

where $\lambda$ represents longitude, $\theta$ represents latitude, and $\triangle \theta=40^{\circ} . T_{e q}(z)$ shown in Figure (2-1) is the typical stratification for the equatorial region used by Liu and Philander (1995). The surface temperature relaxation condition originally proposed by Haney (1971) is used: 


$$
Q_{\text {flux }} / \rho h C_{p}=\frac{1}{\tau}\left(S S T_{\text {ref }}-S S T_{\text {model }}\right)
$$

in which $S S T_{r e f}$ is the initial surface temperature structure, $C_{p}$ is the specific heat capacity, $\rho$ is the density, $h$ is the surface mixed layer depth, and $\tau$ is the relaxation time, 20 days.

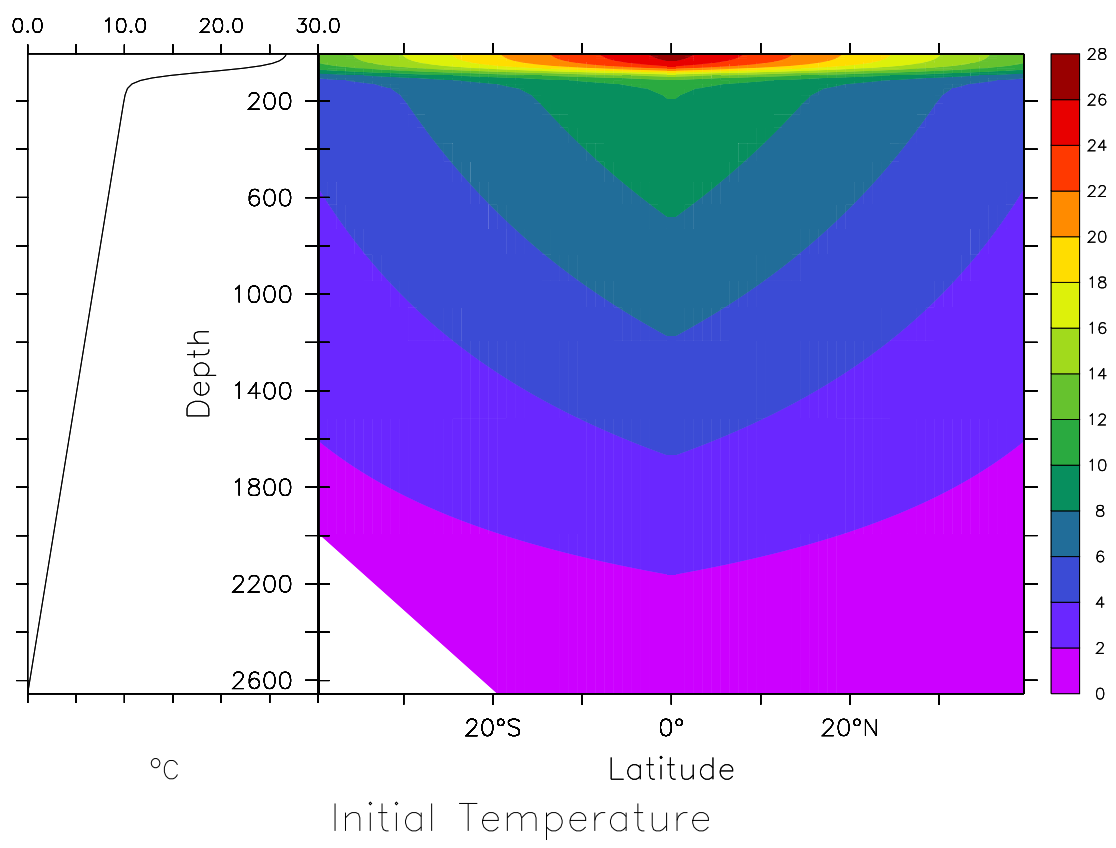

Figure 2-1: Right: The meridional-vertical structure of the initial temperature used for both IH and IL. Left: The vertical profile of the initial temperature at the equator. The initial temperature is zonally uniform and similar to the one used by Liu and Philander (1995). The bowl-shaped initial condition shortens the spin-up time according to the ventilated thermocline theory.

The horizontal mixing is calculated by the Laplacian scheme with eddy viscosity (diffusivity). The coefficients are shown in Table (2.1) for the different simulations. The vertical mixing in the surface mixed layer is also parameterized by the KPP scheme.

The surface wind forcing structure is shown in Figure (2-2). The meridional wind stress, $\tau_{y}$, is zonally uniform. Its meridional structure is shown by the blue line in the upper panel of Figure (2-3). The zonal wind, $\tau_{x}$, has a meridional structure similar to the one originally 
used by Bryan (1987), and is shown by the red line in the upper panel of Figure (2-3). In the zonal direction, $\tau_{x}$ is tapered off over the eastern coastal region within $5^{\circ}$ offshore so that only the along-shore wind component, $\tau_{y}$, exists (Figure (2-3), lower panel).

The model is spun up with the steady wind forcing for 20 years to reach the equilibrium. Liu and Philander (1995) suggested that 20 years are enough for the upper ocean to adjust to equilibrium. The 20th year's outputs are used for analysis.

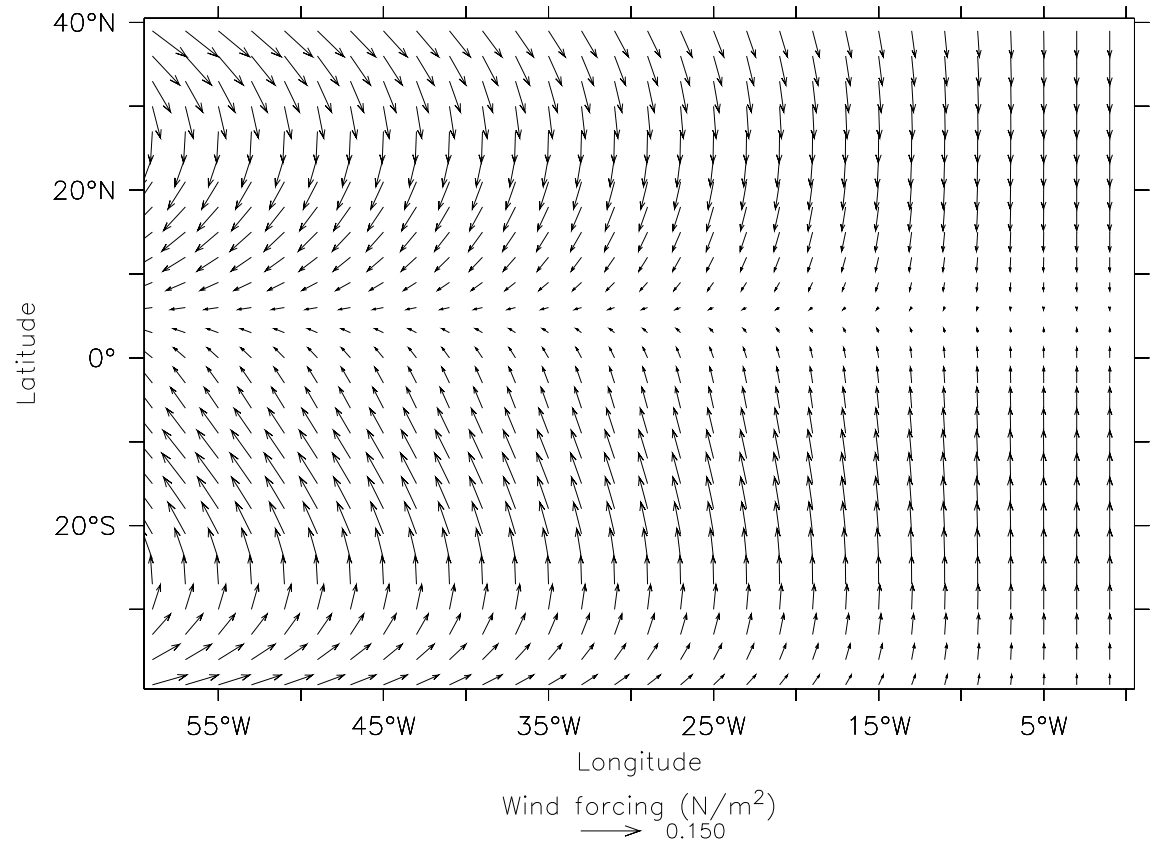

Figure 2-2: The steady surface wind forcing used for IH and IL. There is only along-shore wind stress over the eastern coastal region. 


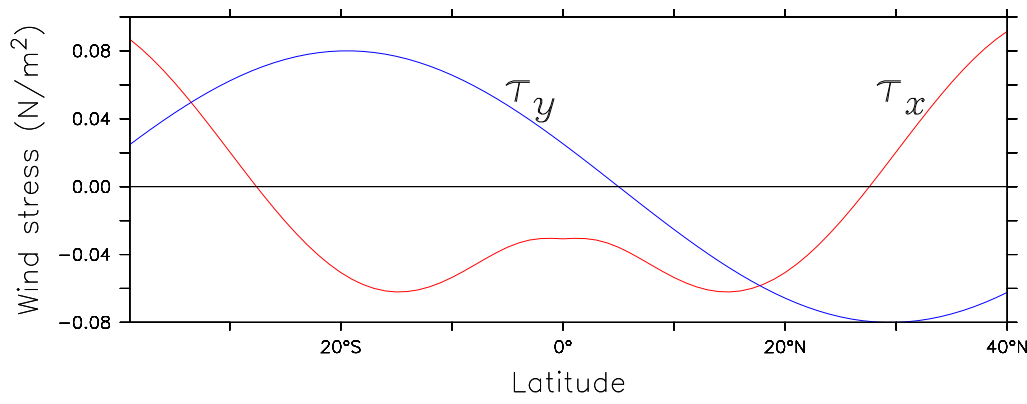

Wind stress along the western boundary (red $\tau_{x}$, blue $\tau_{y}$ )

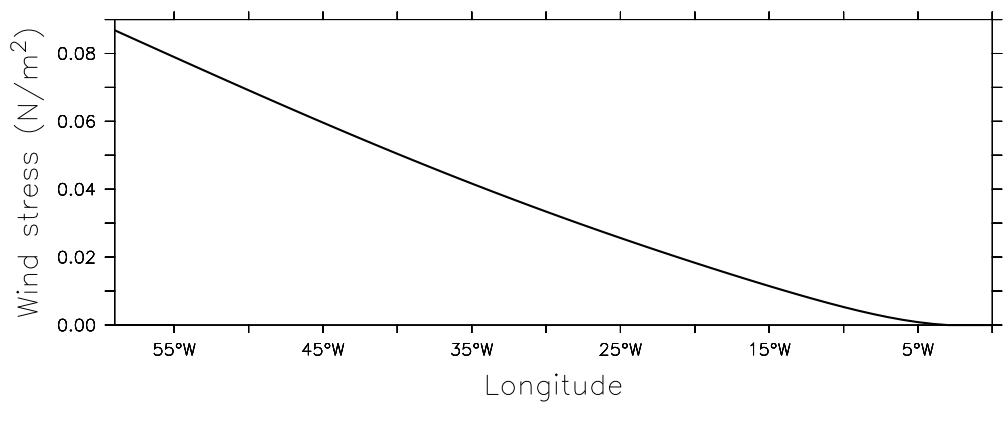

$\tau_{x}$ along the southern boundary

Figure 2-3: Upper panel: The red line shows the zonal wind stress, $\tau_{x}$, along the western boundary, which is similar to the one used in Bryan (1987); the blue line shows the meridional wind stress, $\tau_{y}$, which is zonally uniform. Lower panel: $\tau_{x}$ is damped from 0.09 $\mathrm{N} / \mathrm{m}^{2}$ along the western boundary to zero at the location of $5^{\circ}$ off the coast, and is zero over the $5^{\circ}$-wide coastal region. 


\section{Chapter 3}

\section{Coupled simulations}

In this chapter, I use two coupled simulations to test the hypothesis that better resolving ocean dynamics can improve the SST simulation in the coupled model. The assumption is that more accurate ocean dynamics can be achieved by the ocean model with a higher horizontal resolution. The setups of two experiments, $\mathrm{CH}$ and CL, were discussed in Chapter 2. In the following sections, the structure of SSTs and mean states of coastal currents in the coupled model are investigated. Based on those results, two hypotheses are further proposed to explain the improved SST simulation in $\mathrm{CH}$.

\subsection{Sea surface temperature}

Seo et al. (2006) discussed the general features of the SST difference between CH and CL. They showed that in both experiments the climatological SSTs have similar error distributions compared with the observations, confirming the results of the coupled model simulations by Huang (2004). This systematic warm bias in SST is shared by most of the coupled models, and was discussed by Davey et al. (2002) and Large and Danabasoglu (2006). Although increasing the horizontal resolution does not totally eliminate these biases, it improves the SST pattern over the equatorial and the SWA upwelling region compared with 
observations.

The upper-left panel in Figure(3-1) shows the SST difference between the CL experiment and the observations; the upper-right panel shows the SST difference between $\mathrm{CH}$ and the observations, and the lower panel shows that between $\mathrm{CH}$ and CL. The equatorial SST pattern is improved in $\mathrm{CH}$ with respect to $\mathrm{CL}$ all over the SWA coastal region protruding into the South Atlantic interior. The western patterns are relatively unchanged. This result implies that in $\mathrm{CH}$ the zonal SST gradients are more realistic, and the SST over the SWA upwelling region is improved by reducing the warm bias. However, the bias is not eliminated but only reduced by increasing the horizontal resolution. This implies that other factors, such as surface atmospheric clouds and winds, are also involved in the process. 

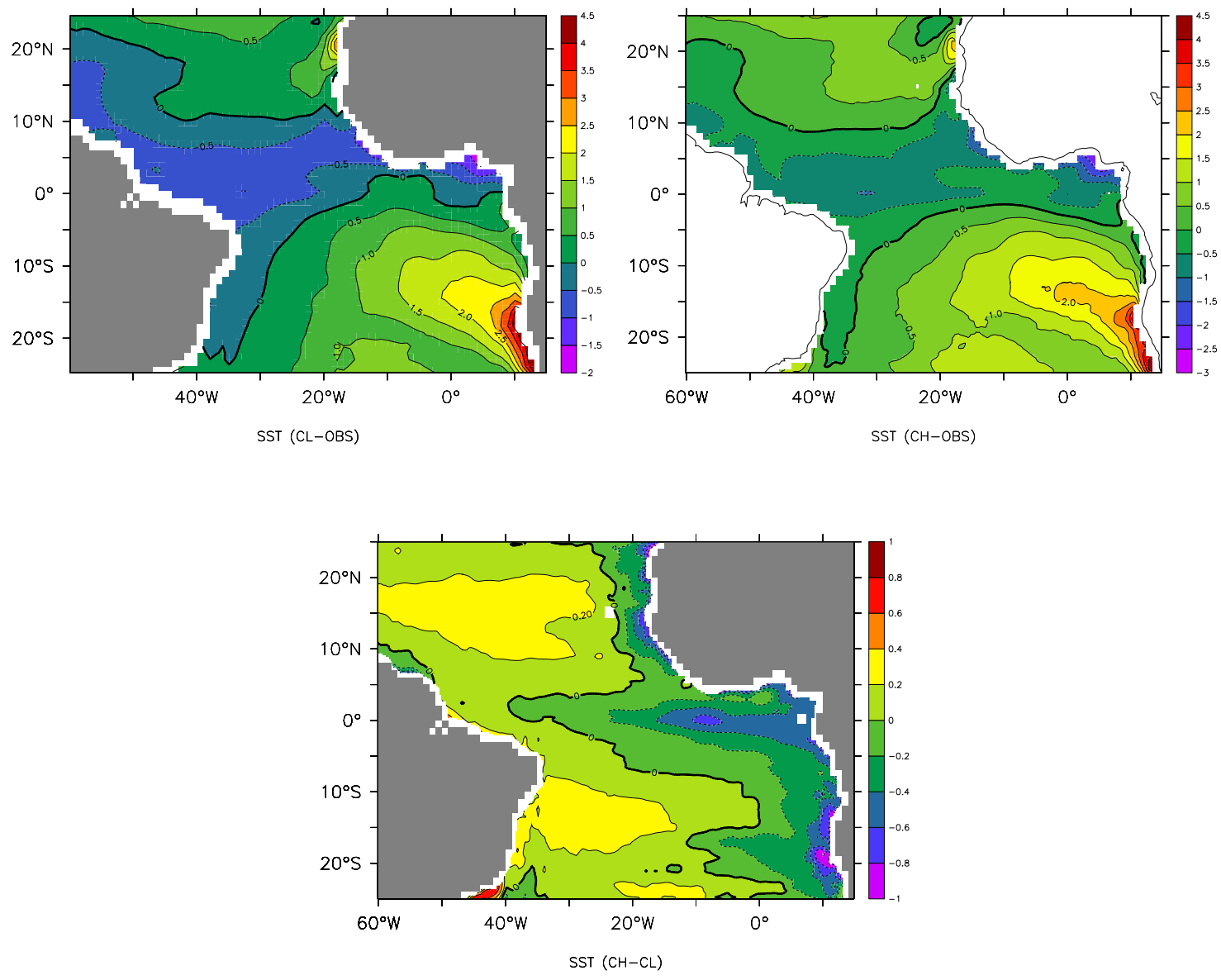

Figure 3-1: The SST differences between CL and observations (upper left), CH and observations (upper right), and $\mathrm{CH}$ and $\mathrm{CL}$ (lower). Both $\mathrm{CH}$ and CL has systematic warm biases over the SWA coastal region. $\mathrm{CH}$ has a maximum of $30 \%$ reduction of the warm bias along the coast. Although increasing the horizontal resolution from $1^{\circ}$ to $1 / 4^{\circ}$ improves the coastal SST simulation, the systematic warm bias is not totally removed, which means airsea interactions, especially the positive feedback between the stratus clouds and SST, are also important. 


\subsection{Possible mechanisms}

Warm biases are reduced by increasing the horizontal resolution in the ocean model, which means the ocean component plays a role in producing biases. Several possible causes responsible for the warm bias are proposed in previous investigations including the problematic representation of the abrupt topographic change along the coast in the spectral atmosphere model, deficiencies of stratus clouds simulation along the coastal upwelling region in the atmospheric boundary layer, and the coastal upwelling (Mechoso et al. 1995; Large and Danabasoglu 2006). The representation of the abrupt topographic change is not the cause of the improvement of the SST simulation here because the land configurations are the same in both $\mathrm{CH}$ and CL. As a result, three main potential mechanisms responsible for the reduction are left: the net heat flux into the ocean might be reduced as a result of the positive feedback between SST and stratus clouds, the surface wind stress along the coast might be changed by the remote atmospheric forcing and be increased in $\mathrm{CH}$, the coastal upwelling might be altered by the increased horizontal resolution of the ocean model and more entrainment cooling occuring in $\mathrm{CH}$. In the following discussion, I show that first two mechanisms can be eliminated, and a more detailed investigation is needed to test the last one.

\section{2a. Net surface heat flux}

Stratus clouds block the short wave radiation into the ocean. The deficiency of stratus clouds leads to more spurious incident short wave radiation into the sea surface and to a warm SST anomaly. It occurs in most coupled climate models where the coarse resolution is used for both the ocean and the atmosphere (Large and Danabasoglu 2006). It is hard to judge which is the cause and which is the effect because of the positive feedback between SST and stratus clouds. In CH and CL, the resolution and physical parameterizations in the atmosphere model are unchanged, but the stratus clouds coverage can still be different 
because of the different SST. The positive feedback mechanism states that cooler SST is more favorable for the stratus clouds formation, which means that stratus clouds over SWA region should be generated more in $\mathrm{CH}$ than in CL because of the cooler coastal SST in $\mathrm{CH}$. More stratus clouds block more short wave radiation and lead to less net surface heat flux into the ocean.

However, as shown in Figure (3-2), the net surface heat flux is larger in CH than in CL, which means that the stratus clouds mechanism is not the reason for the warm bias in this experiment, otherwise the net surface heat flux ought to be smaller in $\mathrm{CH}$ according to the aforementioned argument. $\mathrm{CH}$ has cooler SST over SWA region, so that the temperature difference between the ocean surface and the atmospheric boundary layer is larger in $\mathrm{CH}$ than in CL. As a result, the net surface heat flux is also larger in $\mathrm{CH}$ than in CL according to the air-sea interaction mechanism whose first order approximation is shown by equation (2.2).

To summarize, the stratus clouds mechanism can not explain the reduction of the warm bias over SWA coastal region in this experiment.

\section{2b. Alongshore wind stress}

The wind stress along the coast could be the reason for the warm bias reduction. The wind blows on the surface of the ocean, producing the surface Ekman transport in the upper marine boundary layer. The integrated surface Ekman transport is to the right of the surface wind stress in Northern Hemisphere and to the left in Southern Hemisphere. The divergence of the surface Ekman transport generated by the curl of the surface wind stress causes the cold water to upwell from the subsurface to decrease the SST. When the sidewall boundary appears, the surface wind stress can produce the upwelling only through the along-shore component because of the discontinuity introduced by the side-wall boundary. Along the boundary, the upwelling mass transport is linearly related to the along-shore component of the surface wind stress. As a result, the upwelling is changing according to 


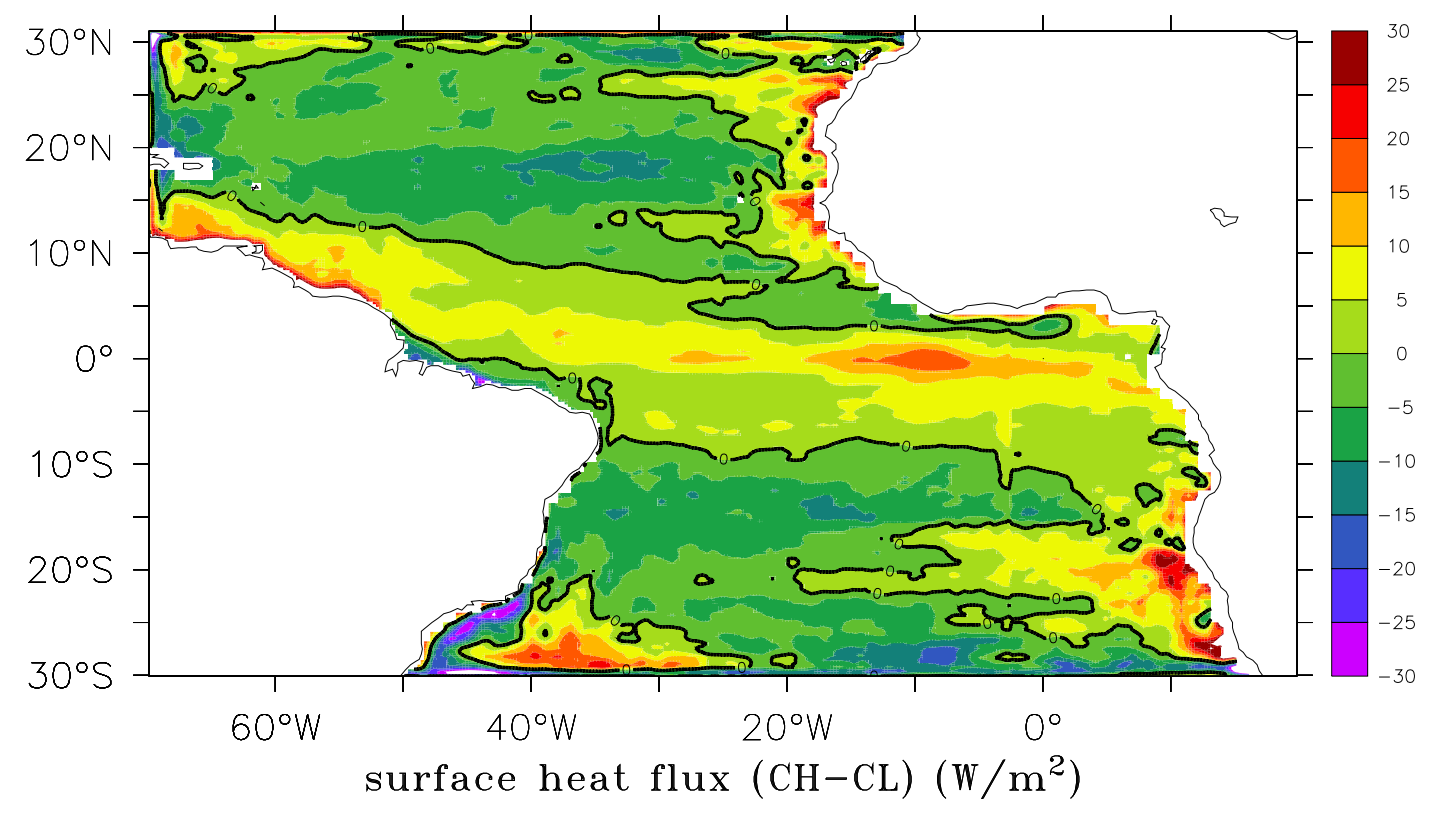

Figure 3-2: The difference of the net surface heat flux between $\mathrm{CH}$ and CL. Positive values mean the ocean in $\mathrm{CH}$ gains more heat than that in CL. 
changes in the wind.

One hypothesis is that the wind stress along the coast might change through remote forcing when the horizontal resolution in the ocean model changes. If the surface wind stress is more upwelling favorable in $\mathrm{CH}$ than in $\mathrm{CL}$, the warm bias reduction might be explained by the wind mechanism, then we should further find the reason for the changed wind. This hypothesis is proved incorrect in this coupled simulations. Figure (3-3) shows the amplitude and the orientation of the wind stress along the coast. In the region south of $15^{\circ} \mathrm{S}$, the wind stress orientation is the same for $\mathrm{CH}$ and $\mathrm{CL}$ but the amplitude is smaller in $\mathrm{CH}$, which means the along-shore wind is more upwelling favorable in CL than in $\mathrm{CH}$ over that region. In the region north of $15^{\circ} \mathrm{S}$, the wind amplitude is the same but the orientation appears to be more upwelling favorable in CL again. These discrepancies are not sufficient to produce a different upwelling, and the warms bias reduction in $\mathrm{CH}$ can not be explained by the wind stress mechanism.

\section{2c. Coastal oceanic processes}

I now investigate the role of ocean dynamics on determining the warm biases.

Previous studies by Mechoso et al. (1995) and by Large and Danabasoglu (2006) inferred that the warm bias might be caused by the underestimated cooling produced by coastal upwelling. Assuming the subsurface water temperature is unchanged, more coastal upwelling means more water transported from the subsurface to the surface. The upwelling in SWA coastal region is mostly fed by the South Equatorial undercurrent (SEUC) (Furue et al. 2007), which is driven by Tropical Instability Waves (TIWs) through an eddy-mean flow interaction mechanism (Jochum and Malanotte-Rizzoli 2004). Jochum and MalanotteRizzoli (2004) showed that the coarsely resolved ocean model can not simulate the TIWs very well, so that the spurious diffusion in the coarse resolution model leads to a weak SEUC. If the subsurface transport by the SEUC decreases, the coastal upwelling decreases accordingly. Figure (3-4) shows that the SEUC is indeed weaker in CL than in CH. How- 

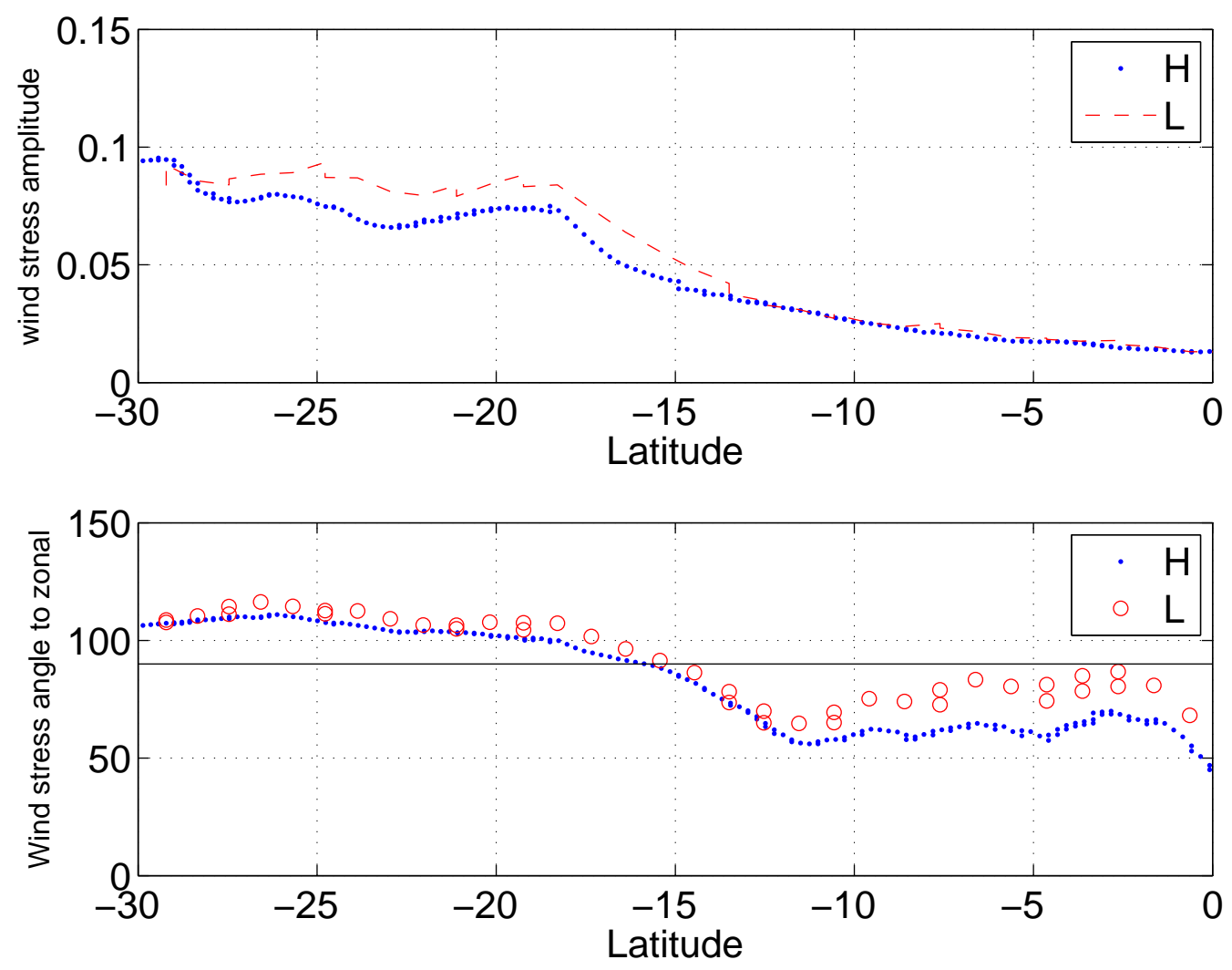

Figure 3-3: The wind stress amplitude (in $\mathrm{N} / \mathrm{m}^{2}$, upper panel) and orientation (in degrees, lower panel) along the SWA coast in $\mathrm{CH}$ (blue) and in CL (red). The black line in the lower panel is $\pi / 2$ representing south-to-north direction. Both the amplitude and the orientation show that the wind stress along the coast is more upwelling favorable in CL. It is hard to quantify the wind-induced upwelling as the coast line representation is different in $\mathrm{CH}$ and CL, especially when grid noise is involved. But qualitatively, the discrepancies are not sufficient to produce the different upwelling in $\mathrm{CH}$ and $\mathrm{CL}$. 

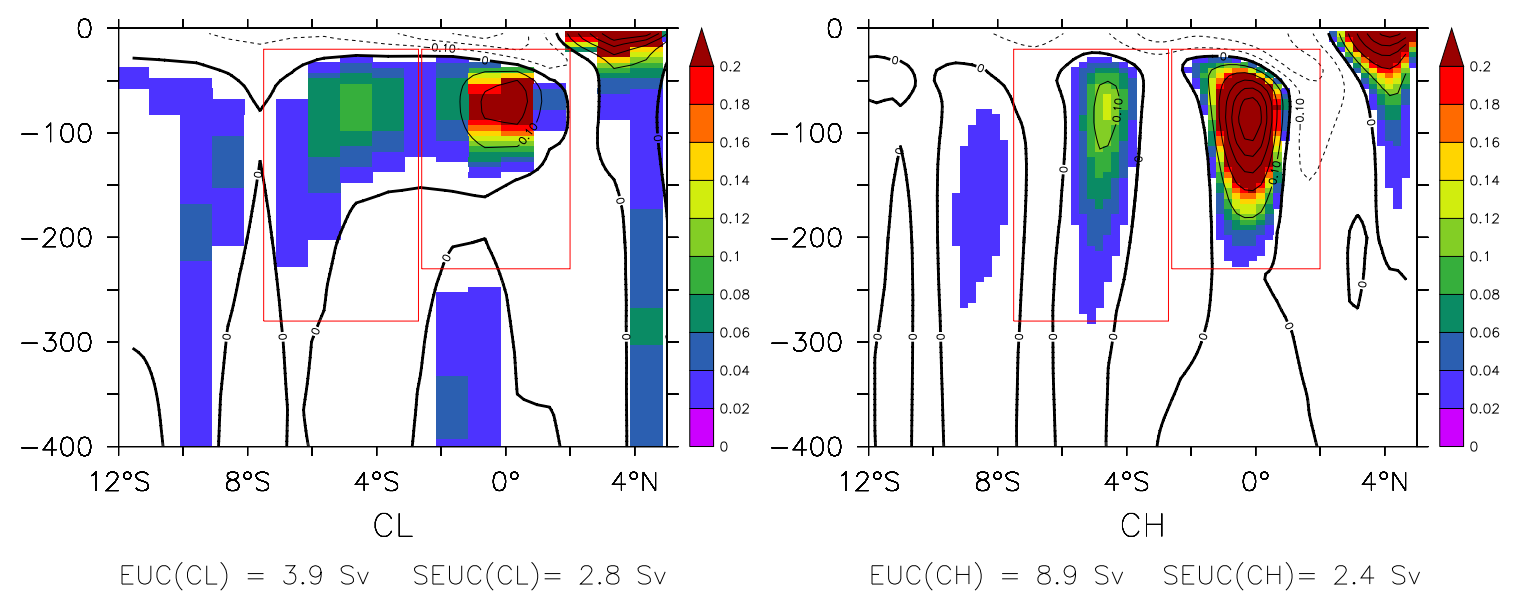

Figure 3-4: The cross section of the zonal velocity along $0^{\circ} \mathrm{E}$ in $\mathrm{CH}$ (right) and $\mathrm{CL}$ (left). The vertical axis is the depth and the horizontal axis is the latitude. In both panels, the shaded areas show eastward velocity, and the color bar is in units of $\mathrm{m} / \mathrm{s}$. The transport by EUC (distinguished by left box in each figure) and SEUC (right box in each figure) is calculated and shown by numbers (in unit of $\mathrm{Sv}, 1 \mathrm{~Sv}=10^{6} \mathrm{~m}^{3} / \mathrm{s}$ ) below the figure. The SEUC is weaker in CL than in $\mathrm{CH}$, but the transport by SEUC is smaller in $\mathrm{CH}$ than in $\mathrm{CL}$ as CL has a wider SEUC. The EUC is stronger in $\mathrm{CH}$ than in CL, but the westward transport is also stronger in $\mathrm{CH}$, which balances the extra onshore flow.

ever, the integrated eastward mass transport by the SEUC is larger in CL than in CH because the SEUC is weaker but broader in CL. It means that we can not simply use the stronger SEUC mechanism to explain the warm bias reduction. I also calculated the net transport for a box-region in SWA area from the eastern, northern and southern boundaries, but found the transport is very sensitive to how the box-region is chosen (figure not shown). Other approaches are needed.

The coastal upwelling is driven by the surface wind stress. The SEUC might be just a responder and act as a feeder to support the upwelling. Under conditions with an upwelling favorable wind, the horizontal coastal currents consist of an offshore Ekman drift in the surface layer fed by onshore undercurrents in the subsurface layer, a surface equatorward along-shore jet and a subsurface undercurrent in the opposite direction. The left panel of 
Figure (3-5) shows the zonal velocity at $400 \mathrm{~km}$ off the coast averaged meridionally over the region between $4 \mathrm{~S}$ and $15 \mathrm{~S}$. In terms of the structure, both $\mathrm{CH}$ and $\mathrm{CL}$ show a coastal current with the surface offshore Ekman drift fed by the onshore undercurrent. In terms of the amplitude, the mean offshore transport in the surface layer is comparable in $\mathrm{CH}$ and in CL as shown in the left panel of Figure (3-5), but the onshore transport in the subsurface layer is greater in CL than in $\mathrm{CH}$. The extra onshore current in $\mathrm{CL}$ downwells and is carried offshore. Figure (3-3) shows that the along-shore component of the surface wind is also comparable in both cases. Given the same surface wind condition, the amount of water upwelled from the subsurface layer will be the same in both $\mathrm{CH}$ and CL. The right panel of Figure(3-5) shows that along shore coastal current has a structure of a equatorward surface jet and a poleward undercurrent which are setup by coastal Kelvin waves. They also exhibit the same amplitude and structure.
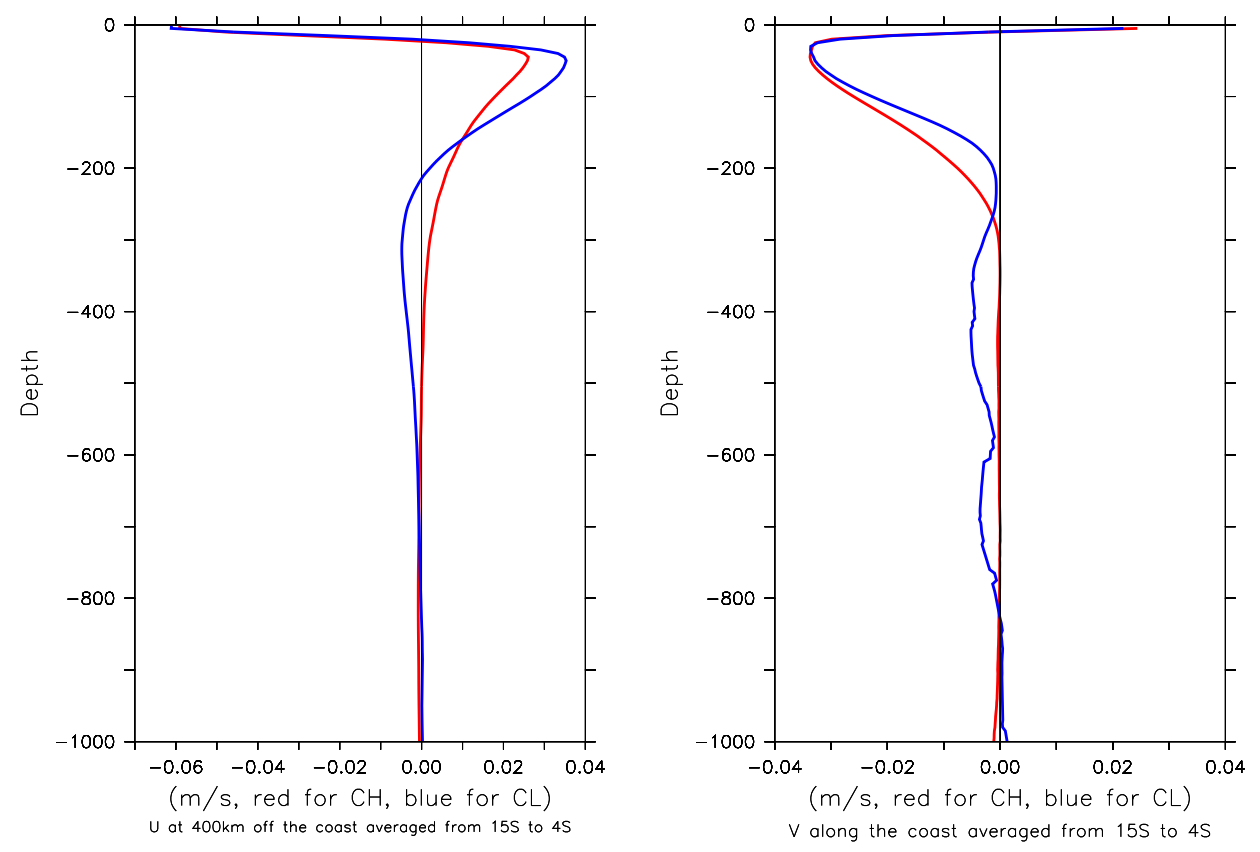

Figure 3-5: Left panel: The zonal velocity at $400 \mathrm{~km}$ off the coast meridionally averaged over the region between $4 \mathrm{~S}$ and $15 \mathrm{~S}$ in $\mathrm{CH}$ (red) and $\mathrm{CL}$ (blue). Right panel: The alongshore velocity averaged in $2^{\circ}$ - wide coastal region between $4 \mathrm{~S}$ and $15 \mathrm{~S}$. 
With the same onshore and offshore flow, $\mathrm{CH}$ and $\mathrm{CL}$ should have the same upwelling if the coastal processes are resolved. Figure(3-6) shows the vertical structures of the mean vertical velocity averaged along the coast between $15^{\circ} \mathrm{S}$ and $4^{\circ} \mathrm{S}$. They exhibit a mixed structure of the first and the second baroclinic mode with upwelling in the upper layer and downwelling in the lower layer. The most significant discrepancy, however, is that both the upwelling and downwelling velocities are much stronger in $\mathrm{CH}$.

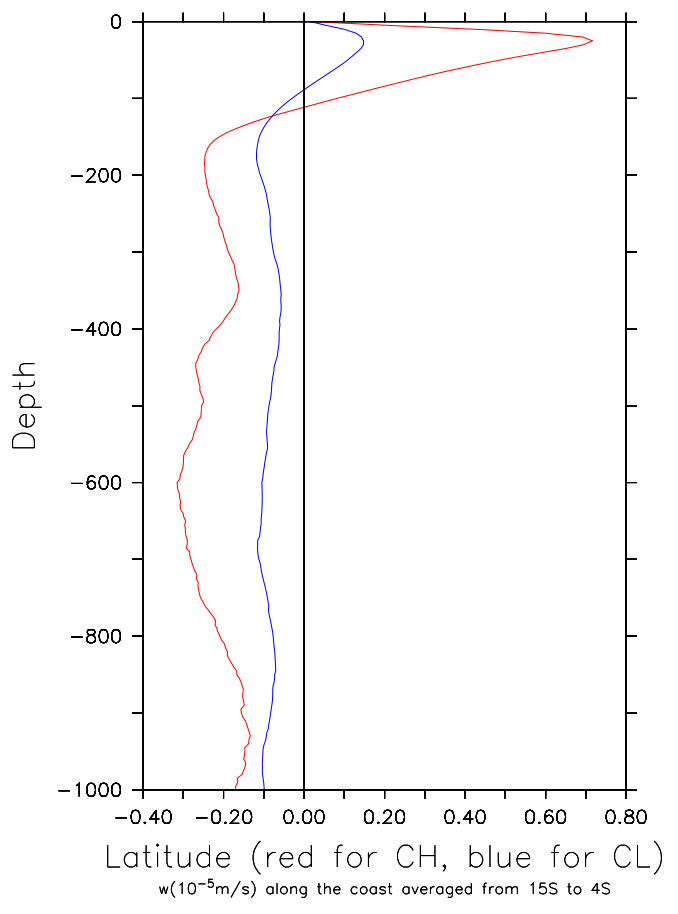

Figure 3-6: The vertical velocity in the eastern-most grid cell at $15 \mathrm{~S}$ (red for $\mathrm{CH}$ ). In the upper ocean, the vertical velocity is much stronger in $\mathrm{CH}$ than in CL.

Figure (3-7) shows the vertical velocity at 30m near the coast along $10 \mathrm{~S}$. The eastern-most grid cell contains the strongest upwelling, the boundary layer determined by the decay scale of the vertical velocity is narrower in $\mathrm{CH}$ than in $\mathrm{CL}$. The coastal processes are different in models with different horizontal resolutions. It leads to the first hypothesis that the misrepresentation of the local response of the coastal boundary layer to the surface wind forcing in coarse resolution simulations may be responsible for the surface warm 


\section{bias.}

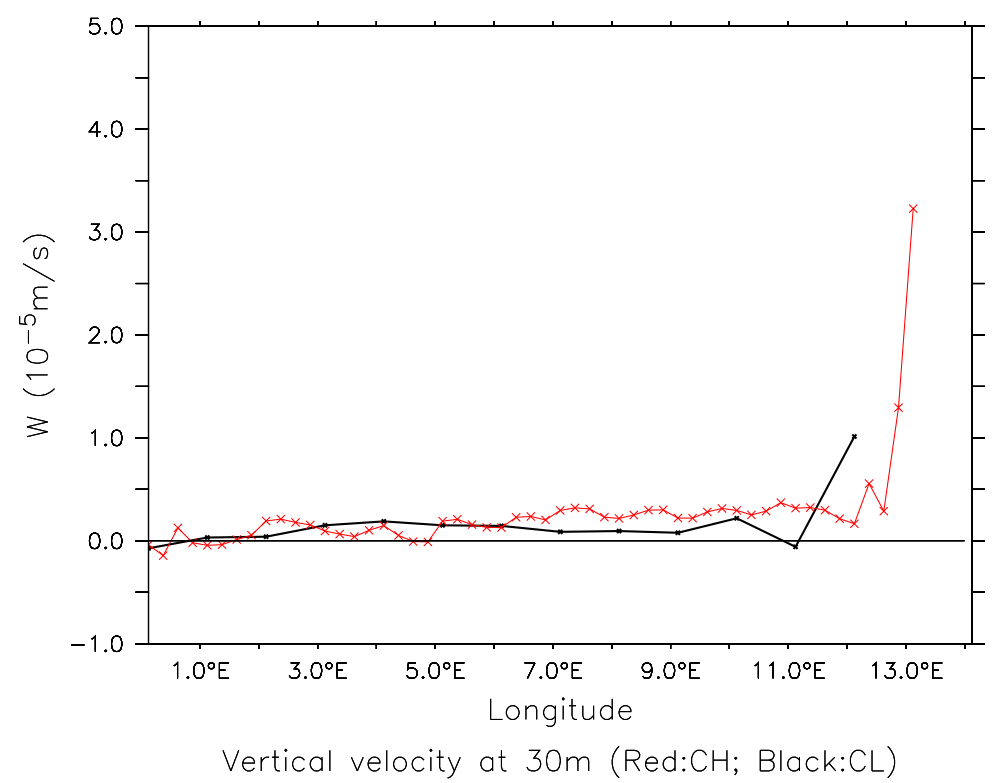

Figure 3-7: The vertical velocity at 30m depth over the coastal region along $10 \mathrm{~S}$ in $\mathrm{CH}$ (Red) and CL (Black). The eastern-most grid cell contains the most significant difference. The vertical velocity is much stronger in $\mathrm{CH}$ than in CL.

However, direct calculation of the upwelling over the SWA coastal region shows different results. We calculated the integrated upward transport at the base of the mixed layer in $\mathrm{CH}$ and $\mathrm{CL}$ for the region comprised between $0^{\circ} \mathrm{E}$-coast in longitude and $15^{\circ} \mathrm{S}-4^{\circ} \mathrm{S}$ in latitude. The upwelling is about $4 \mathrm{~Sv}$ in $\mathrm{CH}$ but only $1.5 \mathrm{~Sv}$ in $\mathrm{CL}$. Most of the upwelling takes place in the near coastal region, within $3^{\circ}$ offshore, which is consistent with the results shown in Figure (3-7). However, excluding the coastal strip from the calculation reduces the upwelling to $1.6 \mathrm{~Sv}$ in $\mathrm{CH}$ and to $1.0 \mathrm{~Sv}$ in $\mathrm{CL}$, which means that the upward transport in the $3^{\circ}$ wide coastal region is $2.4 \mathrm{~Sv}$ in $\mathrm{CH}$ and $0.5 \mathrm{~Sv}$ in $\mathrm{CL}$. This is not consistent with the argument based on the linear and steady state theory discussed above. Wave activities can also be important considering that the coastal undercurrent is established by successive arrivals of coastal Kelvin waves which are excited by the discontinuity of the surface jet at 
the boundaries of the forced region (Yoon and Philander 1982). Mesoscale eddies propagate westward and Kelvin waves come from equatorial regions. Their propagation might have remote influences on the upwelling. It leads to our second hypothesis that the nonlinear and unsteady mesoscale activities over the coastal region are misrepresented in the coarse resolution simulation and may be responsible for the underestimated upwelling in the coastal region, and that the Kelvin wave along the coast might play an important role in mesoscale activities.

\subsection{Uncertainties}

It is important to show that the SST simulation over the SWA coastal region is improved in one regional coupled model by simply increasing the horizontal resolution in the ocean component, or by better resolving the ocean dynamics. However, the coupled model setup used in this experiment leads to several uncertainties that prevent to draw conclusions about what is the mechanism responsible for the improved SST. The first one is related to inconsistent coastlines. As shown in Figure (3-8), the $1^{o}$ coastline has different shape with the $1 / 4^{o}$ one, which introduces the uncertainty that the SST changes might come from the highly resolved coastline, or from the reduced level of grid noises in a narrow coastal region.

The second uncertainty is about the bathymetry. Figure (3-9) shows the bathymetries used by $\mathrm{CH}$ and $\mathrm{CL}$, which are different in both depth and slope. This is caused by a bathymetry filter that is used by ROMS to ensure the numerical stability. The bottom slope in the coastal region plays an important role in altering coastal currents, and an improved bottom slope might lead to the better resolved coastal SST. As the amount of vertical levels in $\mathrm{CH}$ and $\mathrm{CL}$ are the same, the shallow bathymetry in $\mathrm{CH}$ means higher vertical resolution in the upper layer. The improved resolution in both the horizontal and vertical direction introduces the uncertainties that SST might be improved by the higher vertical resolution in the ocean model rather than the horizontal resolution. As a result, associated mechanisms 
are uncertain, and we can not distinguish them only by analyzing the coupled model output.

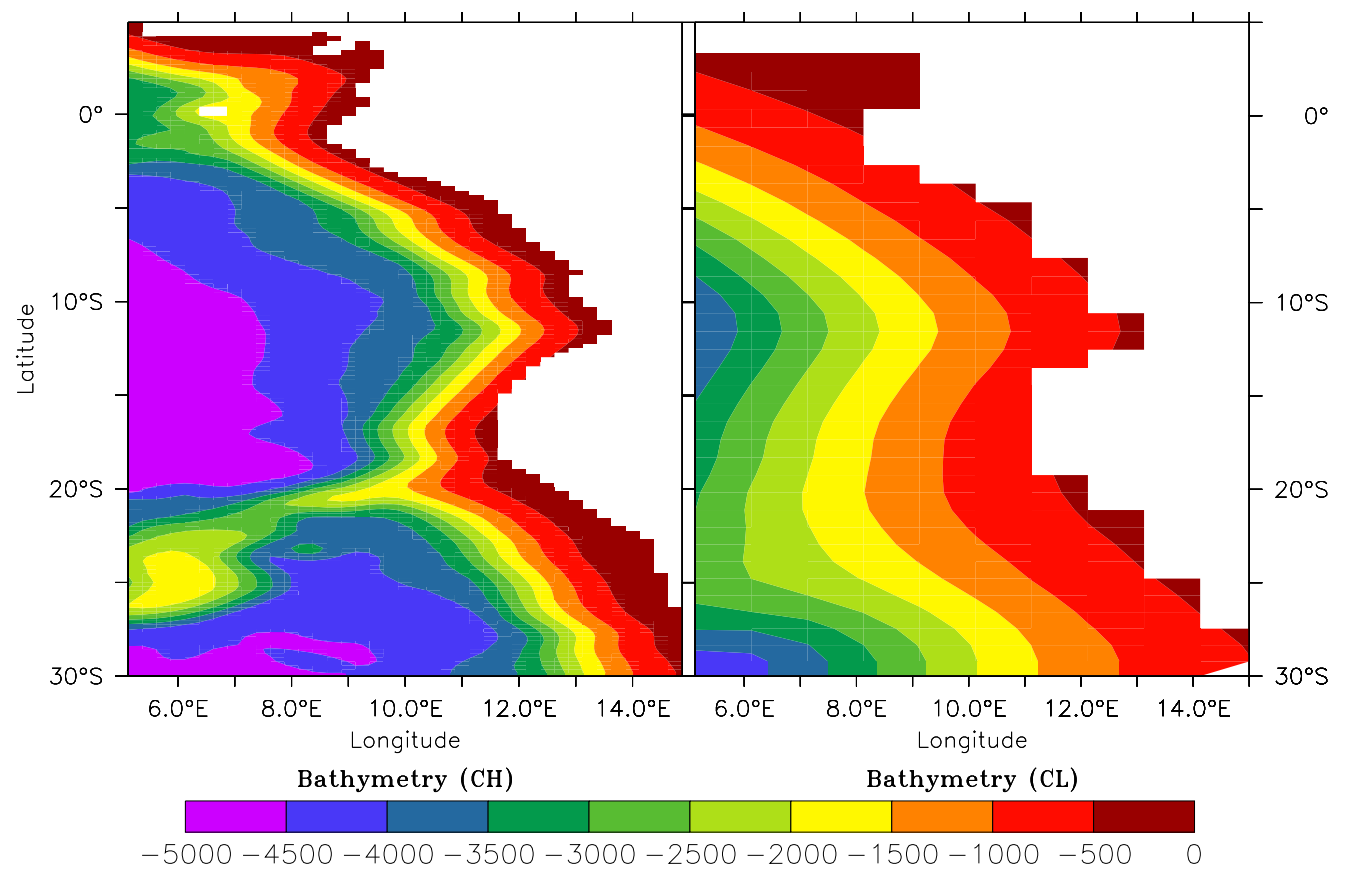

Figure 3-8: Bathymetries used by $\mathrm{CH}$ (right) and CL (left). Notice the differences of coastlines and coastal bathymetries between $\mathrm{CH}$ and $\mathrm{CL}$.

\subsection{Summary}

In this chapter, using the model output given by Seo et al. (2006), I showed that increasing the horizontal resolution in the regional coupled model can improve the SST simulation over the equatorial and coastal regions. Both the high $\left(0.25^{\circ} \times 0.25^{\circ}\right)$ and the coarse $\left(1^{o} \times 1^{o}\right)$ resolution can produce the correct mean structure of the coastal currents, but the coarse resolution has more noise along the coastal boundary. Based on the analysis of the coastal upwelling and the coastal boundary layer, two hypotheses are proposed: 1) the local response of the coastal boundary layer to the surface wind forcing is misrepre- 


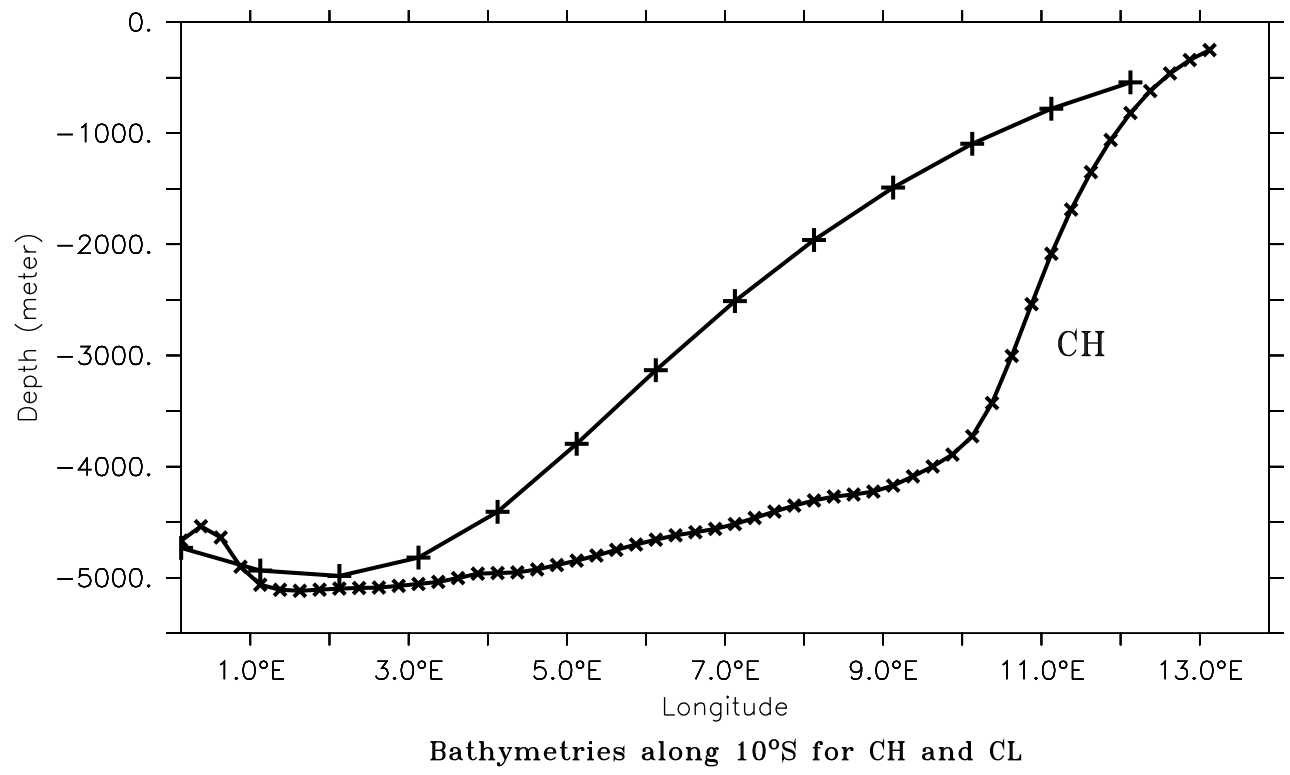

Figure 3-9: One example of the different bathymetry used by $\mathrm{CH}$ and $\mathrm{CL}$. The discrepancy is very large and possibly affects the coastal currents.

sented by the coarse resolution, which causes the surface warm bias; 2) mesoscale eddies are not well simulated in the coarse resolution simulation which further leads to the underestimated coastal upwelling. The first hypothesis has been tested in this study and will be discussed in the following chapters. In order to quantify and distinguish the different dynamical processes, while avoiding uncertainties produced by the irregular coastline and different bathymetries, I will use idealized simulations to investigate the coastal dynamics focusing on the coastal boundary layer. A theoretical review of the coastal dynamics and the analysis of idealized simulations will follow in the next two chapters. 


\section{Chapter 4}

\section{A two dimensional coastal upwelling model}

Results of coupled model simulations show that the eastern-most grid cell in the ocean model contains the most significant differences between $\mathrm{CH}$ and $\mathrm{CL}$. The vertical velocity is much stronger in $\mathrm{CH}$ than in $\mathrm{CL}$ along the coast. In the following, I will focus on the coastal dynamics, and use the theoretical analysis as a guide for understanding the model output.

Let's consider an incompressible fluid satisfying the Boussinesq approximation, driven by an along-shore wind in the vicinity of a meridionally-oriented straight coast in a rotating system with Coriolis parameter $f$. As the wind is steady after being turned on and the coast is straight, the meridional variation $\partial / \partial y \ll(\partial / \partial x, \partial / \partial z)$ and can be neglected. The governing equations are then: 


$$
\begin{aligned}
u_{x}+w_{z} & =0 \\
u_{t}+\mathbf{u} \cdot \nabla u-f v & =-\rho_{0}^{-1} p_{x}+A_{H} u_{x x}+A_{V} u_{z z}, \\
v_{t}+\mathbf{u} \cdot \nabla v+f u & =A_{H} v_{x x}+A_{V} v_{z z}, \\
w_{t}+\mathbf{u} \cdot \nabla w & =-\rho_{0}^{-1} p_{z}-\left(\rho / \rho_{0}\right) g+A_{H} w_{x x}+A_{V} w_{z z}, \\
T_{t}+\mathbf{u} \cdot \nabla T & =K_{H} T_{x x}+K_{V} T_{z z},
\end{aligned}
$$

where the suffixes mean partial derivatives. I apply the scaling analysis between dimensional and nondimensional (starred) variables used by Allen (1973).

$$
\begin{aligned}
t & =f^{-1} t^{*}, \\
(u, v, w) & =U\left(u^{*}, v^{*},(H / L) w^{*}\right), \\
(x, y, z) & =L\left(x^{*}, y^{*},(H / L) z^{*}\right), \\
p & =p_{0}-\rho_{0} g H z^{*}+0.5 \rho_{0} g H \alpha \Delta T_{0} z^{* 2}+\rho_{0} U f L p^{*}, \\
\rho & =\rho_{0}-\rho_{0} \alpha \Delta T_{0} z / H+\rho_{0} U f L /(g H) \rho^{*}, \\
T & =T_{0}+\Delta T_{0} z / H+(f U L / \alpha g H) T^{*} .
\end{aligned}
$$

where $\rho_{0}$ is the constant density in the Boussinesq approximation. The scaling relations imply the assumption that the background equilibrium temperature is linear in the vertical direction, and the density depends linearly on the temperature. The nondimensional 
equations becomes (dropping the asterisks):

$$
\begin{aligned}
u_{x}+w_{z} & =0, \\
u_{t}+\varepsilon \mathbf{u} \cdot \nabla u-v & =-p_{x}+E_{H} u_{x x}+E_{V} u_{z z}, \\
v_{t}+\varepsilon \mathbf{u} \cdot \nabla v+u & =E_{H} v_{x x}+E_{V} v_{z z}, \\
\delta^{2} w_{t}+\delta^{2} \varepsilon \mathbf{u} \cdot \nabla w & =-p_{z}+T+\delta^{2}\left(E_{H} w_{x x}+E_{V} w_{z z}\right), \\
T_{t}+\varepsilon \mathbf{u} \cdot \nabla T+S w & =\frac{E_{H}}{\sigma_{H}} T_{x x}+\frac{E_{V}}{\sigma_{V}} T_{z z},
\end{aligned}
$$

The nondimensional parameters are

$$
\begin{gathered}
\varepsilon=U /(f L), S=(\delta N / f)^{2}, N^{2}=g \alpha \Delta T_{0} / H, E_{H}=A_{H} / f L^{2}, \\
E_{V}=A_{V} / f H^{2}, \delta=H / L, \sigma_{H}=A_{H} / K_{H}, \sigma_{V}=A_{V} / K_{V} .
\end{gathered}
$$

where $\varepsilon$ is the Rossby number, $S$ is the stratification number, $N^{2}$ is the buoyancy frequency, $E_{H}$ is the horizontal Ekman number, $E_{V}$ is the vertical Ekman number, $\delta$ is the aspect ratio, $\sigma_{H}$ is the horizontal Prandtl number, and $\sigma_{V}$ is the vertical Prandtl number. Considering the cases with $\delta \ll 1$ and the Rossby number $\varepsilon$ small, the above equations can be simplified to be:

$$
\begin{aligned}
u_{x}+w_{z} & =0, \\
u_{t}-v & =-p_{x}+E_{H} u_{x x}+E_{V} u_{z z}, \\
v_{t}+u & =E_{H} v_{x x}+E_{V} v_{z z}, \\
p_{z} & =T \\
T_{t}+S w & =\frac{E_{H}}{\sigma_{H}} T_{x x}+\frac{E_{V}}{\sigma_{V}} T_{z z},
\end{aligned}
$$

Boundary conditions on the side-wall are no-slip, no-normal flow and no-heat-flux condi- 
tion:

$$
u=v=w=0, T_{x}=0 ; x=x_{E}
$$

where $x_{E}$ stands for the eastern boundary. The surface boundary condition is:

$$
w=u_{z}=0, v_{z}=\tau_{y} ; z=0
$$

\subsection{Vertical velocity and Ekman pumping}

Within the upper surface boundary layer, the vertical variation is much larger than the horizontal variation $\left(u_{x} \sim \mathrm{O}\left(10^{-5} s^{-1}\right), u_{z} \sim \mathrm{O}\left(10^{-3} s^{-1}\right)\right.$ at $10 \mathrm{~m}$ depth next to the coast in IH). For steady state, we can write $(4.15 \mathrm{c})$ with boundary conditions:

$$
\begin{gathered}
u=E_{V} v_{z z}, \\
v_{z}=\tau_{y}(y), z=0, \\
v_{z}=0, z=-h,
\end{gathered}
$$

in which $\tau_{y}(y)$ is the alongshore wind component and a function of $y$ (we do not have $\tau_{x}$ ), and $-h$ is the depth of the base of the mixed layer. Integrating (4.18a) with boundary conditions leads to:

$$
V_{u}=d y \int_{-h}^{0} u d z=E_{V} \tau_{y}(y)
$$

where $V_{u}$ represents the offshore volume transport in the surface Ekman layer. It can be written back in the dimensional form as $V_{u}=\tau_{y}(y) / \rho_{0} f$. It is the offshore volume flux in the upper Ekman layer with a thickness of $O\left(E_{V}^{1 / 2}\right)$, and is fed by the upwelling in the side-wall boundary layer. Also, the total volume transport of the upwelled water can be 
calculated using the vertical velocity at the base of the mixed layer:

$$
V_{w}(x)=d y \int_{x}^{x_{E}} w(x, y) d x
$$

where $V_{w}$ means the volume transport calculated from the upwelling velocity. Outside of the boundary layer, $x \ll x_{e}-\delta x$ where $\delta x$ is the boundary layer width, $V_{u}$ should be identical to $V_{w}$, because no upwelling happens when the curl of the wind stress is zero. The relationship between $V_{u}$ and $V_{w}$ in the idealized model simulation will be shown in the following chapter.

\subsection{Boundary layer during spin-up}

Allen (1973) showed that during the initial spin-up on the time scale of $O\left(E_{V}^{1 / 2}\right)$, the fluid can be regarded as inviscid and the variables can be scaled as:

$$
\begin{aligned}
& t=E_{V}^{-1 / 2} \bar{t} \\
& u=E_{V} u_{0}(x, z, \bar{t})+\cdots, v=E_{V}^{1 / 2} v_{0}+\cdots, w=E_{V} w_{0}+\cdots \\
& p=E_{v}^{1 / 2} p_{0}+\cdots, T=E_{V}^{1 / 2} T_{0}
\end{aligned}
$$

Assuming $E_{V}$ is small

$$
E_{V} \ll O(1)
$$


The governing equations are

$$
\begin{aligned}
u_{0 x}+w_{0 z} & =0, \\
v_{0} & =p_{0 x} \\
v_{0 \bar{t}}+u_{0} & =0 \\
p_{0 z} & =T_{0}, \\
T_{0 \bar{t}}+S w_{0} & =0,
\end{aligned}
$$

It shows that in the meridional direction the leading order is the geostrophic balance. Eliminating $p_{0}, v_{0}$, and $T_{0}$ gives the vorticity equation:

$$
\left(u_{0 z}-S w_{0 x}\right)=0
$$

Introducing the stream function

$$
u_{0}=\psi_{0 z}, w=-\psi_{x}
$$

(4.24) then becomes

$$
\nabla_{S}^{2} \psi=S \psi_{x x}+\psi_{z z}=0
$$

which means that the potential vorticity, $\nabla_{S}^{2} \psi$ is conserved during spin-up. It also shows that there is a side-wall boundary layer with the scale of $\delta_{x}=O\left(S^{1 / 2}\right)$. Its dimensional form, $L_{x}=O(N H / f)$, is the Rossby radius of deformation. We may consider the spinup process setup through coastal Kelvin wave adjustment, which is confined within the boundary layer with the width of $L_{x}$. 


\subsection{Diffusive boundary layers}

After the initial spin-up, the surface coastal jet stops accelerating, and the upwelling is suppressed after the passage of successive Kelvin waves. The diffusivity becomes important on the time scale of $O\left(S E_{H}^{-1}\right)$ (Allen 1973) in the side-wall boundary layer with width of $O\left(S^{1 / 2}\right)$. Then the governing equations for the steady state are:

$$
\begin{aligned}
u_{x}+w_{z} & =0, \\
v & =p_{x}, \\
u & =E_{H} v_{x x}+E_{V} v_{z z}, \\
p_{z} & =T \\
S w & =\frac{E_{H}}{\sigma_{H}} T_{x x}+\frac{E_{V}}{\sigma_{V}} T_{z z} .
\end{aligned}
$$

To the first order, the geostrophic balance is satisfied in the along-shore direction, and the Coriolis force is balanced by the offshore diffusion of the meridional velocity. Depending on the relative magnitude of the horizontal and vertical diffusion, the equations can be simplified into two cases.

In the first case, I consider $E_{H} \gg E_{V}$ and $E_{H} / \sigma_{H} \gg E_{V} / \sigma_{V}$, which means that the vertical viscosity and diffusivity are small and can be neglected. The equations become:

$$
\begin{aligned}
u_{x}+w_{z} & =0, \\
v & =p_{x}, \\
u & =E_{H} v_{x x}, \\
p_{z} & =T, \\
w & =\frac{E_{H}}{\sigma_{H} S} T_{x x},
\end{aligned}
$$


We can write above equations in terms of stream function $\psi$ :

$$
\psi_{z z}+\sigma_{H} S \psi_{x x}=0
$$

which shows that the horizontal boundary layer width is of $O\left(\sigma_{H}^{1 / 2} S^{1 / 2}\right)$. This is the hydrostatic layer (Barcilon and Pedlosky 1967). In the case where the horizontal Prandtl number $\sigma_{H}=O(1)$, there are no major changes in the structure of $\psi$ from the one discussed in previous section where the boundary layer width is $O\left(S^{1 / 2}\right)$.

In the second case, I consider $E_{H} \ll E_{V}$ and $E_{H} / \sigma_{H} \ll E_{V} / \sigma_{V}$, which means that the vertical variation becomes important. The governing equations are

$$
\begin{aligned}
u_{x}+w_{z} & =0, \\
v & =p_{x}, \\
u & =E_{V} v_{z z}, \\
p_{z} & =T, \\
w & =\frac{E_{V}}{\sigma_{V} S} T_{z z},
\end{aligned}
$$

Applying the same algebra as for the first case, the equations can be written in terms of the stream function $\psi$ :

$$
\psi_{z z}+\sigma_{V} S \psi_{x x}=0
$$

which shows that the boundary layer width is $O\left(\sigma_{V}^{1 / 2} S^{1 / 2}\right)$. If we choose the horizontal and vertical Prandtl numbers to be the same, say $\sigma$, the two widths will be identical: $L=$ $\sigma^{1 / 2} N H / f$ in the dimensional form.

Based on the scaling argument, three types of the boundary layer width are obtained: 


$$
\begin{aligned}
L_{d} & =N H / f \\
L_{d H} & =\sigma_{H}^{1 / 2} N H / f \\
L_{d V} & =\sigma_{V}^{1 / 2} N H / f
\end{aligned}
$$

$L_{d}$ is the scale for the inviscid flow adjustment, $L_{d H}$ is the scale when the horizontal diffusion dominates in the heat equation, and $L_{d V}$ is the same as $L_{d H}$ but when the vertical diffusion dominates.

Obviously, there are other side-wall boundary layers depending on the particular consideration of the stratification and side-wall boundary conditions. Stewartson (1957) showed that for homogeneous rotating fluid the boundary layers have a double structure with thickness of $O\left(E^{1 / 4}\right)$ and $O\left(E^{1 / 3}\right)$. Barcilon and Pedlosky (1967) showed that there are three regions in the parameter space of the Ekman number, E, and the stratification number, $\mathrm{S}$, when both of them are small. For weakly stratified cases, the side-wall boundary layer is order of $\left(E^{1 / 4}\right)$ which is analogous the one in homogeneous case; for strongly stratified cases, the side-wall boundary layer has thickness of $O\left(E^{1 / 2}(\sigma S)^{-1 / 4}\right)$ where viscous and buoyancy forces balance; for intermediate cases, the side-wall boundary layer has a hybrid structure but is dynamically dominated by the hydrostatic layer with thickness of $O\left((\sigma S)^{1 / 2}\right)$. Allen (1973) show that a steady coastal current is also confined to a diffusion boundary layer with a thickness of $O\left(\lambda^{-1 / 2}\right)\left(\lambda=E_{V} / E_{H}\right)$. However, the vertical velocity in the diffusion boundary layer discussed by Allen (1973) is too weak to contribute to the vertical mass balance, and also the non-hydrostatic layer $\left(O\left(E^{1 / 2}(\sigma S)^{-1 / 4}\right)\right)$ is too thin to be significant in the vertical mass transport. I did not discuss those cases because I will focus on the vertical mass transport, and the hydrostatic boundary layer is the most important one.

In the following chapter, I will discuss the model's capability in resolving the boundary layer, and corresponding effects on the vertical heat transport. The discussion about the 
heat transport answers the question why increasing horizontal resolution can improve the SST simulation over the SWA coastal region in the coupled model. 


\section{Chapter 5}

\section{Idealized simulations}

Using the idealized forced ocean model, I have done four simulations to address the question why increasing the horizontal resolution can improve the coastal SST simulation: IH, IL, IHH1, and IHH2. The model configuration details are discussed in Chapter 2. The surface wind over coastal region is simplified so as to include only the alongshore component without curl. The wind remains steady after being turned on. The model configurations imply that IH and IL are idealized versions of $\mathrm{CH}$ and $\mathrm{CL}$. The SST comparison between $\mathrm{IH}$ and IL shows the same improvement as is shown in $\mathrm{CH}$ and $\mathrm{CL}$. $\mathrm{IHH}_{1}$ and $\mathrm{IHH}_{2}$ use different horizontal Prandtl number. By comparing their results, the theoretical framework discussed in Chapter 4 is confirmed. In the following sections, the coastal features in $\mathrm{IHH}_{(1,2)}$ are first described, and then followed by the comparison between IH and IL.

\subsection{Results with resolved boundary layers}

In $\mathrm{IHH}_{(1,2)}$, the zonal resolution near the coast is $0.05^{\circ}$ (approximately $5 \mathrm{~km}$ ), which is sufficient to resolve the coastal side-wall boundary layer. Figure (5-1) shows the temperature field with the streamfunction along $12^{\circ} \mathrm{S}$ within the $3^{\circ}$-wide coastal region in $\mathrm{IHH}_{1}$. The isotherms are lifted up towards the side-wall boundary because of the strong upwelling. 
The offshore transport is carried in the upper $25 \mathrm{~m}$. The onshore undercurrent is relatively broad and slow. Three lines indicate three different boundary layers, $L_{d V}, L_{d H}$ and $L_{d}$ from left to right respectively. As $\sigma_{H}=2, L_{d}$ is comparable to $L_{d H}$. While $L_{d V}$ is much larger than the former two because $\sigma_{V}=10$. The structure of the stream function shows the boundary layer width is more comparable to $L_{d H}$, which indicates that the horizontal diffusion/viscosity terms play dominate roles in the steady state.

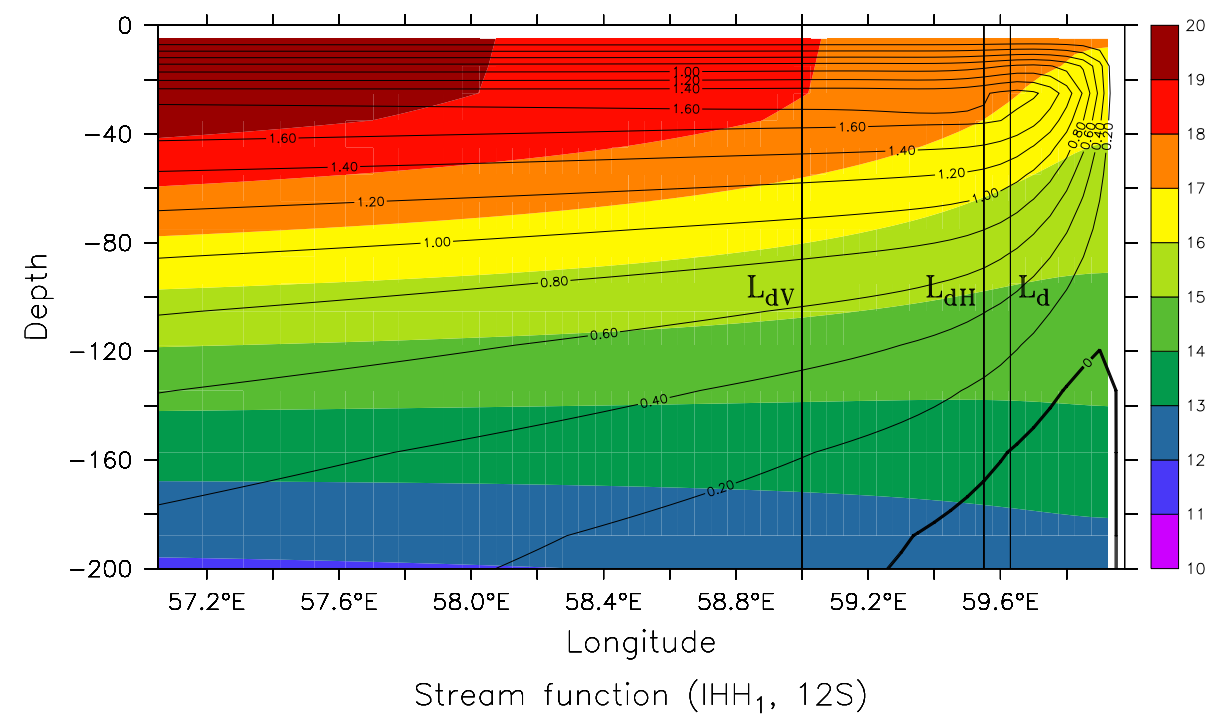

Figure 5-1: The temperature field (shaded) and the stream function (contour) along $12^{\circ} \mathrm{S}$ over 3 degrees offshore in $\mathrm{IHH}_{1}$. Three lines indicate the location of $L_{d V}, L_{d H}$ and $L_{d}$ from left to right. The stream function is calculated using $\psi=\int_{-H}^{z} u d z$, where $-H$ is the bottom depth.

In $\mathrm{IHH}_{2}$, the horizontal Prandtl number is increased from 2 in $\mathrm{IHH}_{1}$ to 5 . We can see from the velocity structures shown in Figure (5-2) that the side-wall boundary layer width is changing with $L_{d H}$. The width is wider in $\mathrm{IHH}_{2}$ than in $\mathrm{IHH}_{1}$. Further examination of heat diffusion term in three directions, $E_{H} T_{x x}, E_{H} T_{y y}$ and $E_{V} T_{z z}$ confirm that the horizontal diffusion is largest and the meridional heat diffusion is negligible (Figure (5-3)).

Given the same surface wind forcing condition, i.e. the same upwelling volume transport 

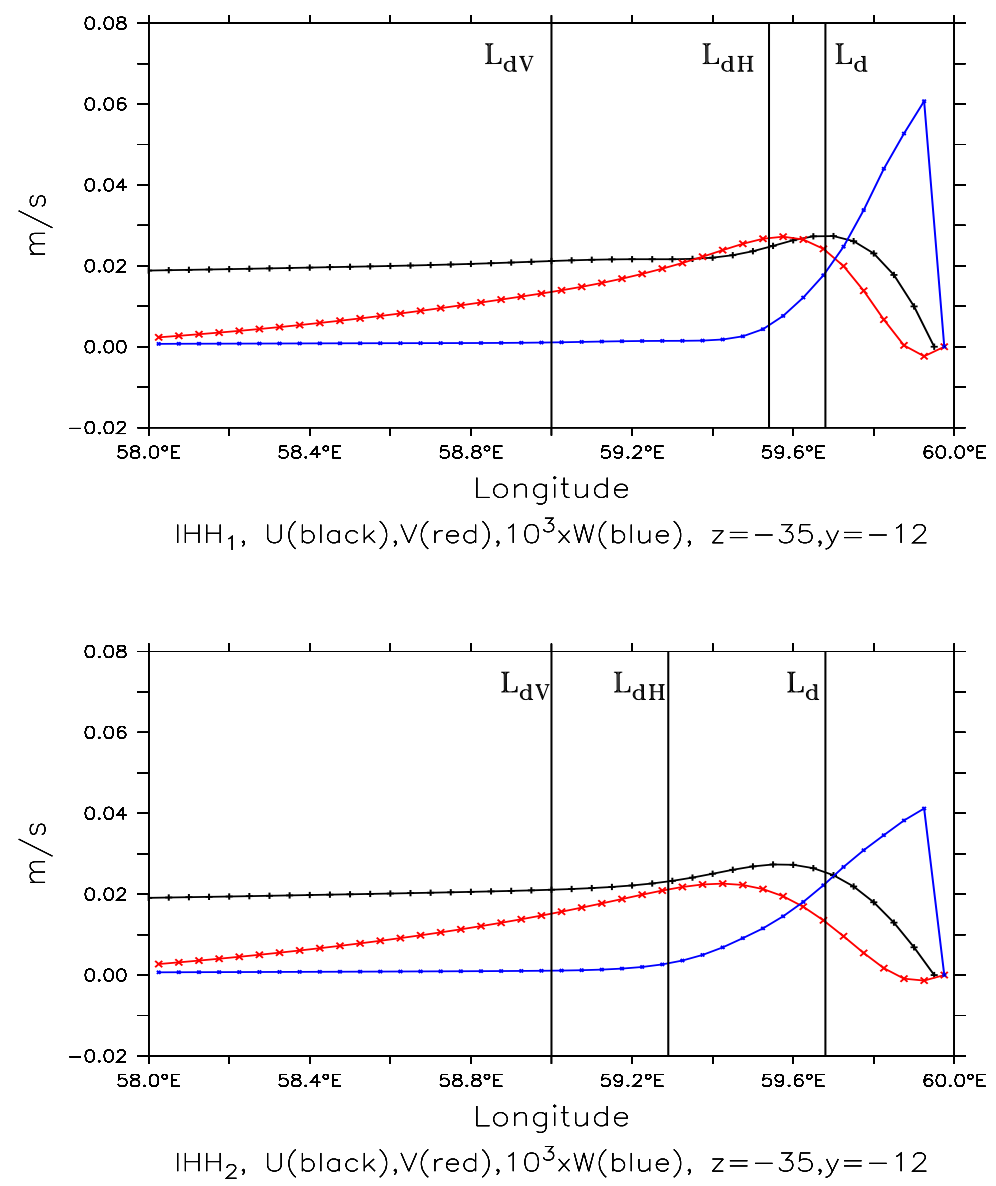

Figure 5-2: Velocity (Black is for u, red for v, and blue for w) structure along $12^{\circ} \mathrm{S}$, at the level of $35 \mathrm{~m}$ deep and 2 degrees offshore. Upper panel is for $\mathrm{IHH}_{1}$ with horizontal Prandtl number $\sigma_{H}=2$, and lower panel is for $\mathrm{IHH}_{2}$ with horizontal Prandtl number $\sigma_{H}=5$. The vertical lines are the same as those in Figure(5-1). We can see that the boundary layer width changes mostly with the horizontal diffusion boundary layer. The frictional boundary layer, which is necessary to satisfy the non-slip condition for the vertical velocity, is not explicitly resolved because the model is hydrostatic. This layer is extraordinarily thin so that it carries negligible fraction of the vertical mass flux (Pedlosky 1987) and is not important in this study. 


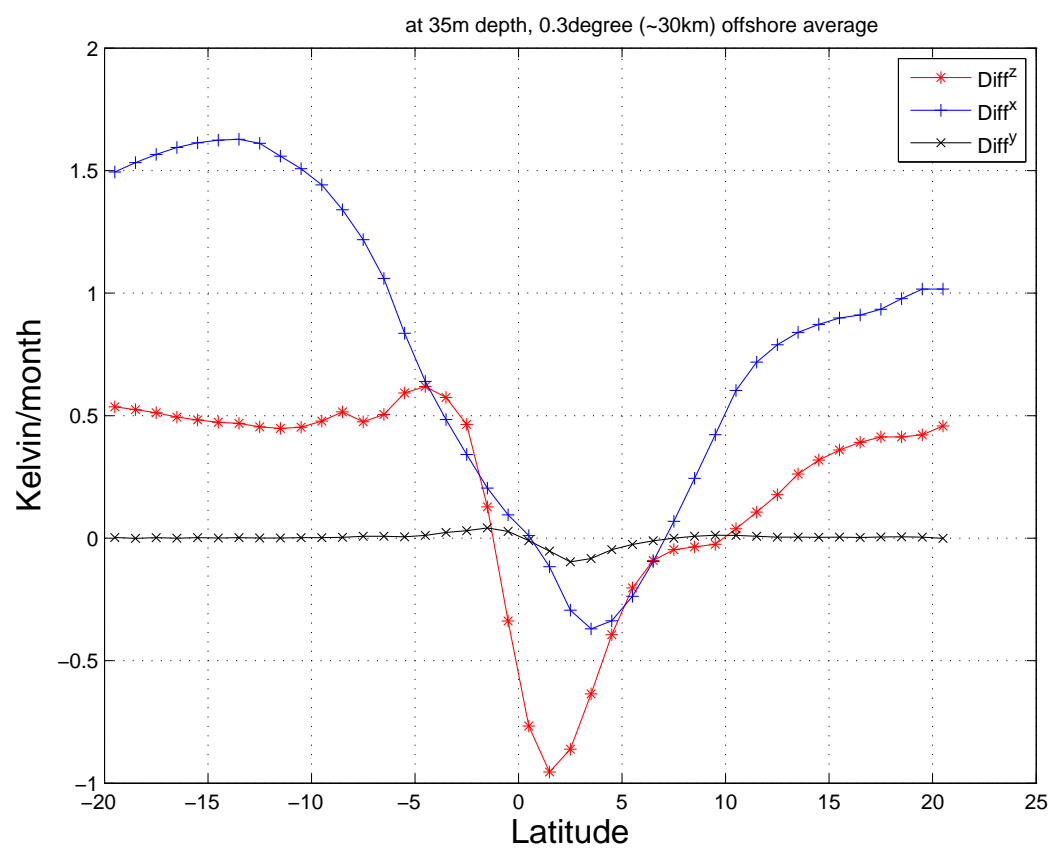

Figure 5-3: Diffusion terms in zonal (blue), meridional (black) and vertical directions below the mixed layer, $35 \mathrm{~m}$ in $\mathrm{IHH}_{1}$. They were calculated every timestep by the model itself. In the region away from equator, the zonal diffusion term dominates.

within the side-wall boundary layer, the vertical velocity will decrease following the increase of the width of the boundary layer. It is clear in Figure (5-2) that the maximum of the vertical velocity within the boundary layer decreases from $6 \times 10^{-5}$ to $4 \times 10^{-5}$ as $L_{d H}$ increases. The changes also affect the SST clearly. As shown in Figure (5-4) the SST is cooler in the case with narrower boundary layer $\left(\mathrm{IHH}_{1}\right)$.

The above results show that $I H H_{1,2}$ are able to catch the basic coastal dynamics. I will refer to them as 'true' results.

\subsection{Results with unresolved boundary layers}

As is shown in Figure(5-5), the SST over the coastal region is cooler in IH than in IL, which is consistent with what happens in the coupled model. Given same atmospheric conditions, 


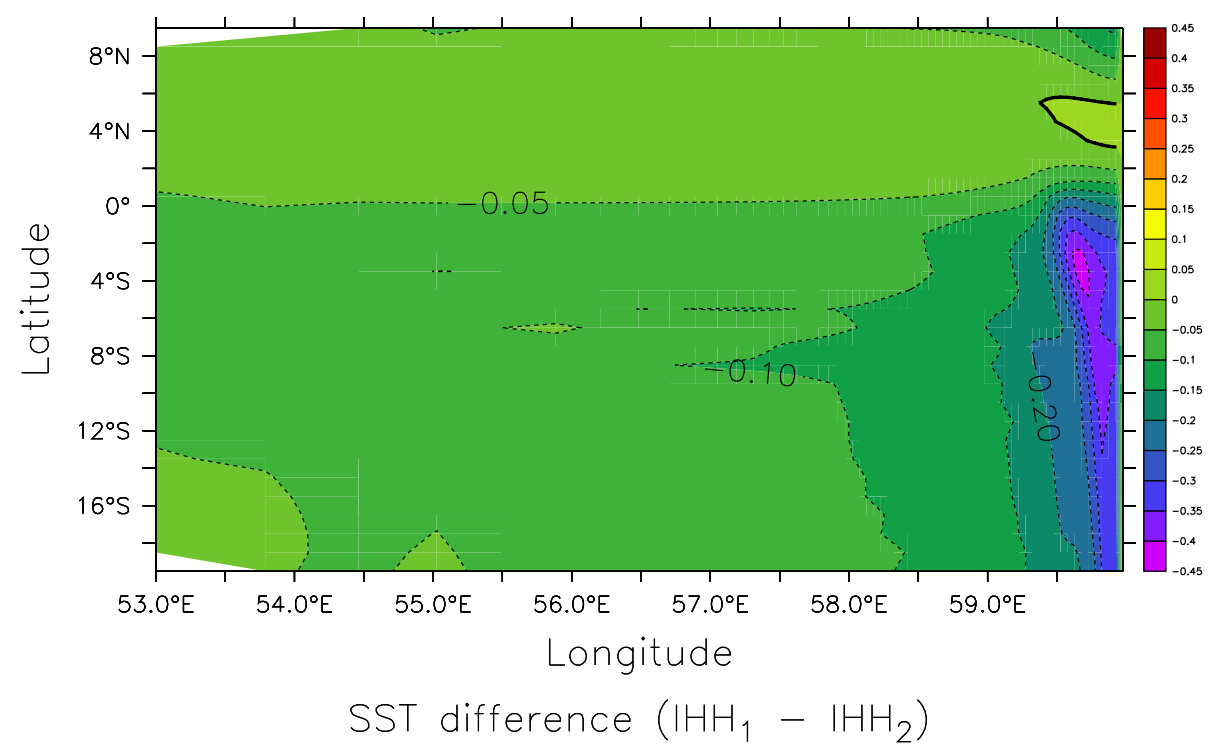

Figure 5-4: The SST difference between $\mathrm{IHH}_{1}$ and $\mathrm{IHH}_{2}$. The negative value means SST is cooler in $\mathrm{IHH}_{1}$.

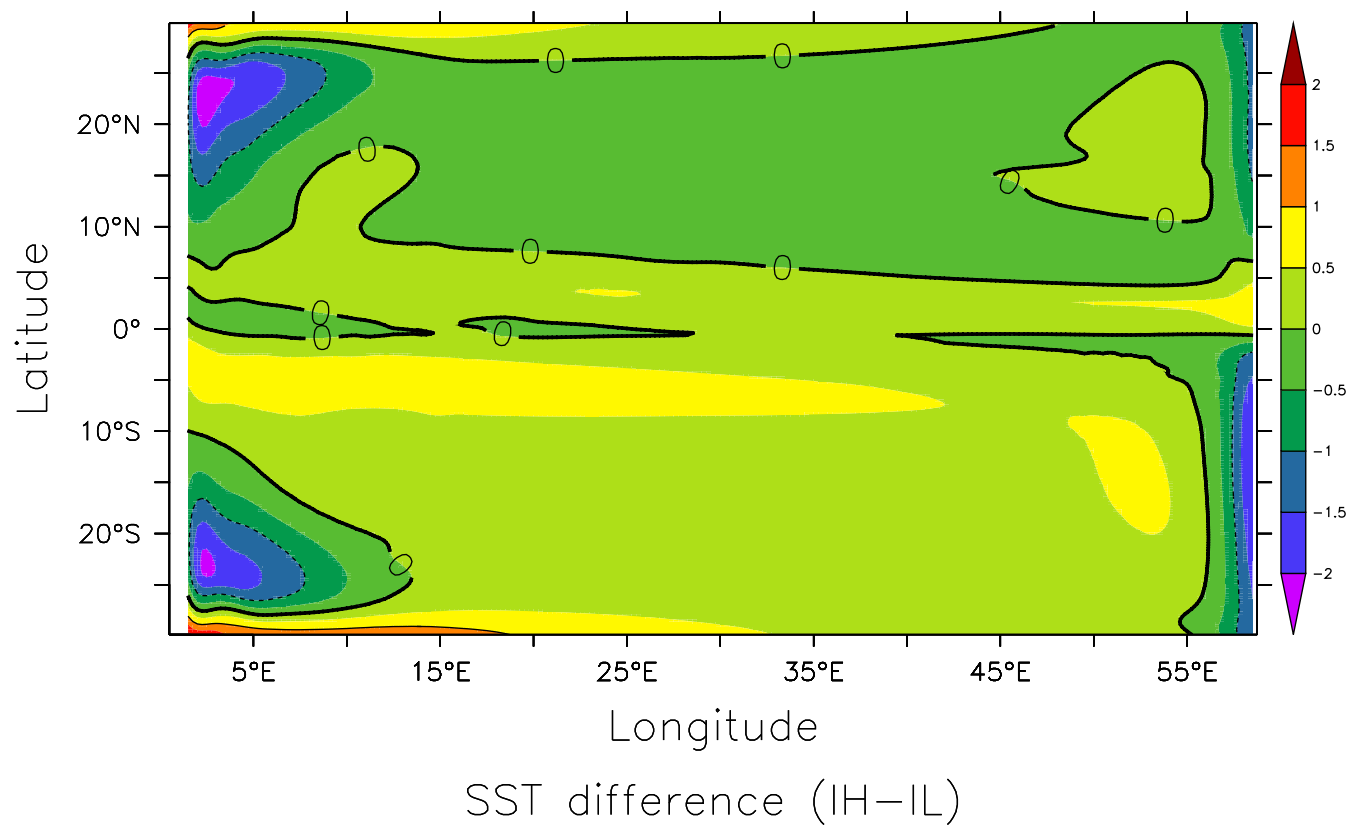

Figure 5-5: The temperature difference (IH - IL). The coastal region SST decreases in the high resolution simulation. 


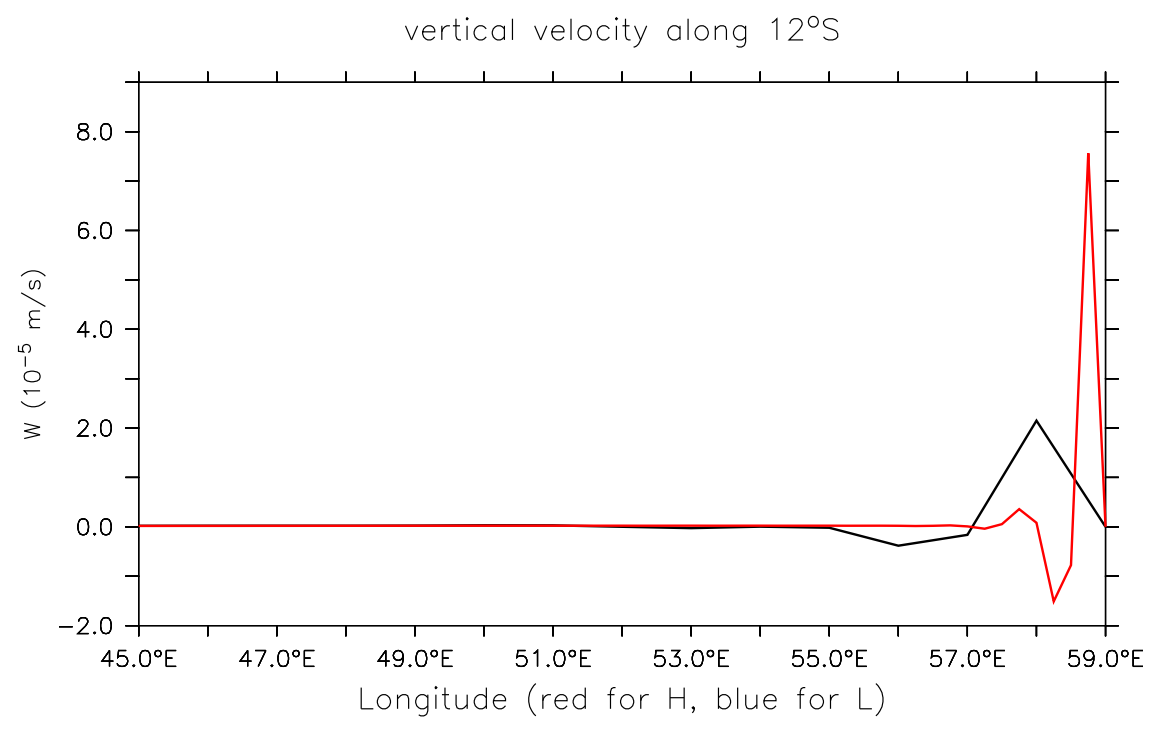

Figure 5-6: The vertical velocity along $12^{\circ} S$ near the eastern boundary. The upwelling rate in the high resolution simulation is intenser than in low resolution. Both 0.25 and 1 resolution are unable to well resolve the coastal activities as the upwelling is active in only one grid (IL) or 1.5 grid (IH).

this result suggests that the coastal SST can be improved by resolving ocean dynamics without involving the air-sea coupling.

Unlike $0.05^{\circ}$ used in $I H H_{1,2}$, both $0.25^{\circ}$ and $1^{\circ}$ used in IH and IL are incapable of resolving the coastal activity (Figure (5-6)), where the first baroclinic Rossby radius of deformation is approximately $40 \mathrm{~km}$ at latitude of $12^{\circ} \mathrm{S}$. As a result, almost all the upwelling is confined in the eastern-most grid (Figure (5-6)). The vertical volume transport, or mass transport, over the coastal region should be identical as long as the wind stress is the same according to the relation between (4.19) and (4.20). It is also true in IH and IL as shown in Figure (5-7).

According to (4.20), the accumulated vertical volume transport starting from the eastern boundary at a certain level can be rewritten as:

$$
V_{w}(x, z)=\rho \int_{y 1}^{y 2} d y \int_{x}^{x_{E}} w(x, y, z) d x
$$




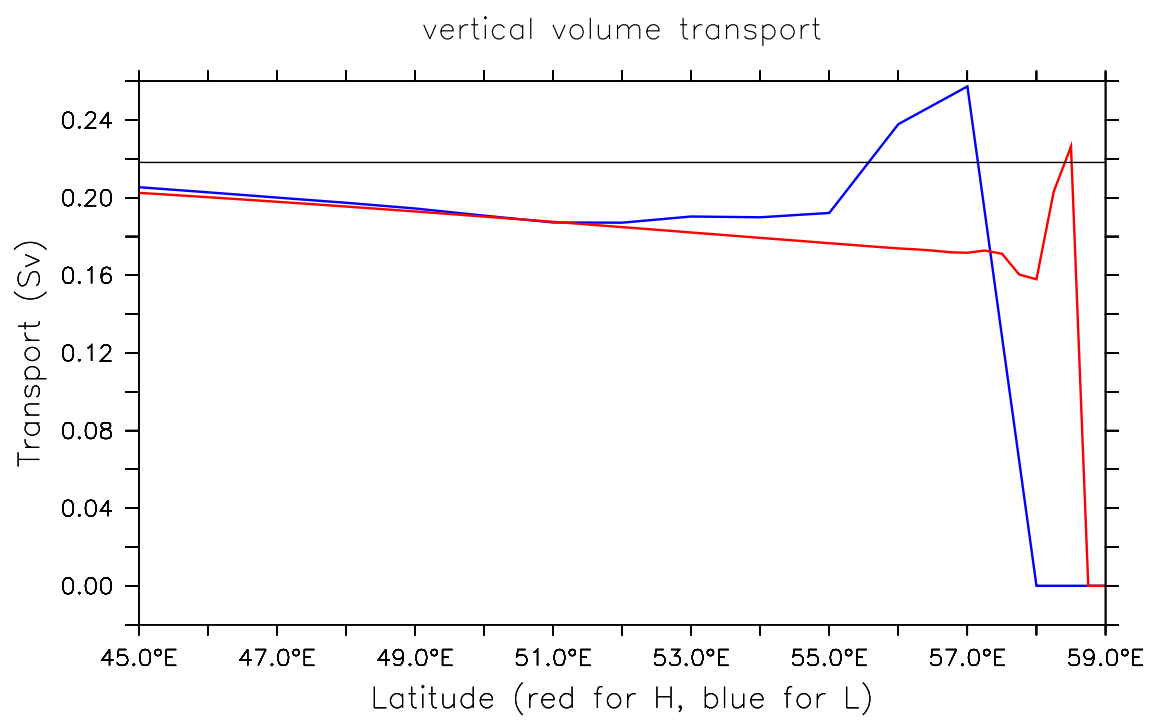

Figure 5-7: The integration of the vertical transport at $25 \mathrm{~m}$ near the eastern boundary. $y_{1}=11^{\circ} S$, $y_{2}=12^{\circ} \mathrm{S}, z=25 \mathrm{~m}$ for IH(red) and IL(blue). The black line shows the surface Ekman transport, $U_{E}=\tau_{y} / \rho_{0} f$. We can see that far away from the boundary grid, the vertical volume transport is the same for both IH and IL, which means the vertical transport is determined by the surface wind induced the Ekman transport (black line).

in which, $x_{E}$ is the longitude of the eastern boundary, $y_{1}$ and $y_{2}$ is the southern and northern boundaries of the integration area. As shown in (Figure (5-7)), the vertical volume transports are not affected by the horizontal resolution, and are the same in both IH and IL in the region beyond the eastern boundary layer. Also, the edge grid carries most of the transport for both coupled and idealized cases as shown in the Figure (3-7 and 5-7). Using the relationship of equation (4.20) and (4.19), we can get

$$
V_{w}=V_{u}=\tau_{y} / \rho_{0} f \Rightarrow w=\frac{\tau_{y}}{\rho_{0} f} \frac{1}{\Delta x}
$$

where $\Delta x$ is the zonal resolution. Figure (5-6) shows that the magnitude of the vertical velocity along the boundary in IH is four times larger than in IL because $\Delta x_{I L}=4 \times \Delta x_{I H}$. Figure (5-8) shows the stream function and the temperature in IH and IL. The boundary layer, if exists, is much broader in IL than in IH. Consequently, the upwelling velocity in 

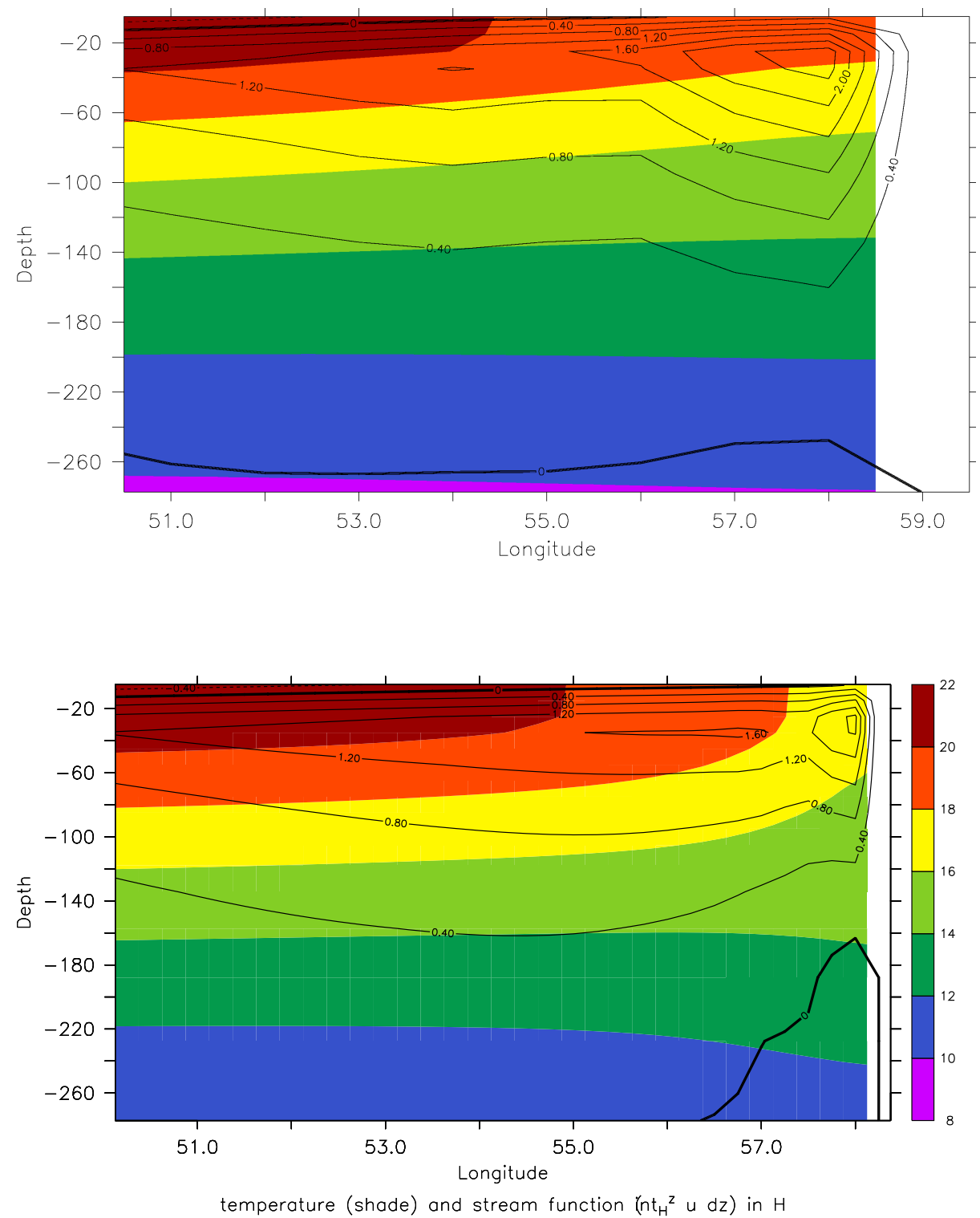

Figure 5-8: The contour shows stream functions $\psi$ calculated by $\int_{-H}^{z} u\left(x, z^{\prime}\right) d z^{\prime}$ along $12^{\circ} S$ in the region 10 degrees offshore for IL (upper) and IH (lower). The color contour is the temperature. When close to the boundary, the vertical transport upwells the subsurface cold water to the surface. The vertical transport of $2.4 \mathrm{~m}^{2} / \mathrm{s}$ is carried in the eastern most grid, and the same for both IH and IL. 
IH is stronger than in IL, so that the isotherms are more titled near the boundary. The discrepancy in the SST field suggests that the resolution also affects the heat transport although the volume transports are the same.

\subsection{The effect of horizontal resolution on the heat trans- port equation.}

If the coarse resolution model can resolve the boundary layer dynamics but with an artificially stretched boundary layer, then the horizontal length scale $L$ will significantly change. If the stretched length, $L_{S}$, is $\mathbf{n}$ times larger than the 'true' value, $L_{s}=\mathbf{n} L$, then the new horizontal Ekman number becomes, $E_{H S}=E_{H} / \mathbf{n}^{2}$. It indicates that the stretched boundary layer reduces the relative importance of the horizontal diffusion and increase the relative importance of the vertical diffusion. Under certain conditions, for example $n$ happens to be 3 so that the horizontal diffusion is approximately one tenth of the original one; the vertical diffusion will be dominate in the heat equation. The scaling analysis of the heat equations shows that vertical diffusion terms in IH and IL indeed exceed the horizontal diffusion significantly (Figure(5-9)).

\subsection{Simple relationship between the zonal resolution and the surface cooling}

The horizontal resolution, especially the zonal resolution for meridionally-oriented coasts, affects the coastal boundary layer dynamics. It also changes the heat transport in the coastal side-wall boundary layer, which is indicated in Figure (5-4). The relationship between the zonal resolution and the surface cooling by the upwelling entrainment within the coastal boundary layer is discussed as follows. 

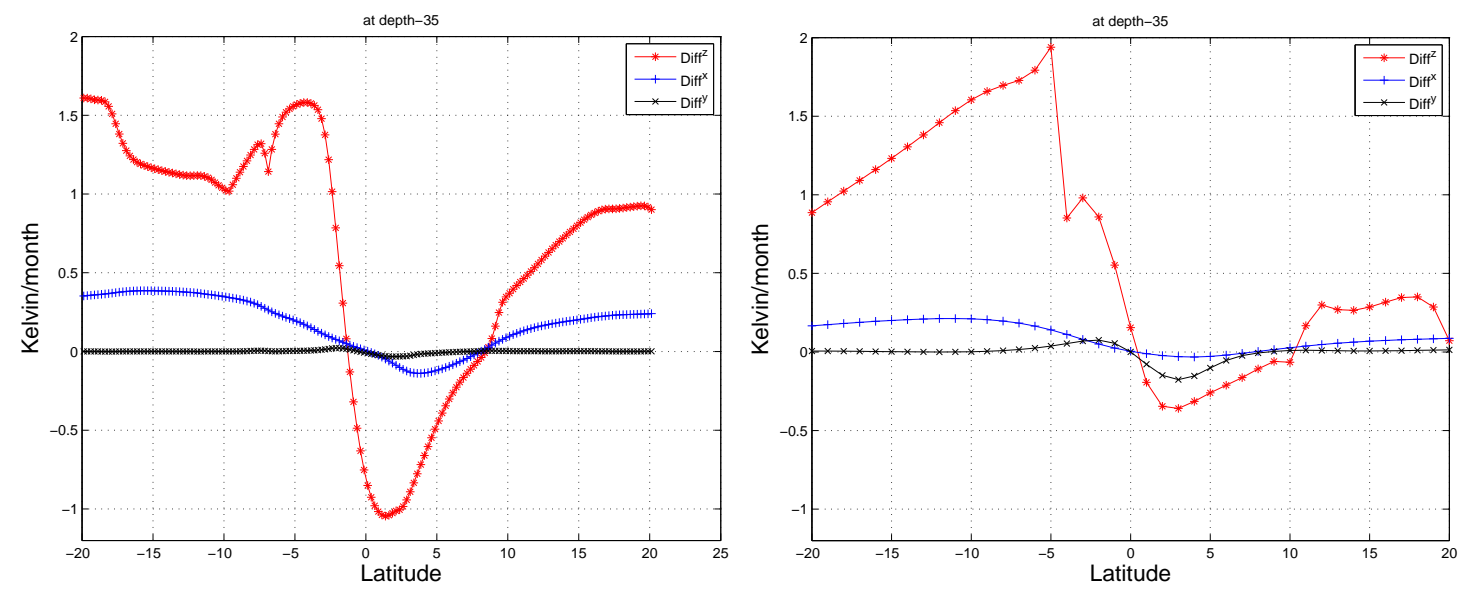

Figure 5-9: Diffusion terms in zonal (blue), meridional (black) and vertical (red) direction below the mixed layer at $35 \mathrm{~m}$ along the coast for IH (left) and IL (right). They are calculated every timestep by the model itself. Almost anywhere, especially in the SWA region $\left(15^{\circ} S\right.$ to $\left.5^{\circ} S\right)$, the vertical diffusion term dominates. Notice that in the north equatorial region, diffusion terms become negative because of the downwelling.

Based on the output of IH and IL, further scaling analysis shows:

$$
\begin{aligned}
& v_{y} \sim O\left(10^{-8}\right),\left(u_{x}, w_{z}\right) \sim O\left(10^{-7}\right) \\
& \left(u T_{z}, v T_{y}\right) \sim O\left(10^{-8}\right), w T_{z} \sim O\left(10^{-6}\right) \\
& \delta \sim O\left(10^{-3}\right), \varepsilon=O\left(10^{-2}\right) \\
& E_{H} \sim O\left(10^{-4}\right), E_{V} \sim O\left(10^{-3}\right)
\end{aligned}
$$

which suggests that we can write (4.5) for the steady state as

$$
w T_{z}=K_{V} T_{z z}
$$

in the dimensional form. Here the vertical advection term is not written in the form of the stratification number $S$, because unlike in Allen (1973), I assume that in the strong upwelling regime the perturbation of the background stratification can be of the same order 
as the background itself. That is, in

$$
T=T_{0}+\Delta T_{0} z / H+T^{\prime}
$$

where $T^{\prime}$ is the temperature perturbation from the linear equilibrium background, and $T^{\prime}$ is of the same order as $\Delta T_{0}$. As a result of (5.5), $S$ is no longer a constant parameter in the heat equation.

A one-dimensional vertical heat equation is obtained in the eastern-most grid. The sidewall boundary layer is thinner than one zonal grid cell, so that it is forced to be the easternmost grid. Variables lose their horizontal structure in the boundary layer. The upwelling velocity becomes the mean value of the upwelling velocity in the 'true' boundary layer, and is inversely proportional to the zonal resolution, $\Delta x$. The diffusion is dominated by its vertical component. Then we can write the heat equation with boundary conditions as

$$
\begin{aligned}
w T_{z} & =\left(K_{V} T_{z}\right)_{z} ; \\
T_{z} & =Q_{0}, z=0 ; \\
T & =T_{b}, z=-\delta ;
\end{aligned}
$$

where $Q_{0}$ represents the surface heat flux condition. Because the scaling is valid below the base of the surface mixed layer, the vertical boundaries are chosen to be $z=0$ at the base of the mixed layer, and $z=-\delta$ at the depth below the base of the mixed layer where the upwelling is weak and broad, and the horizontal diffusion is no longer negligible.

Solving the equation with the boundary conditions gives

$$
T(z)=\frac{Q_{0}}{\exp \left(\int_{-\delta}^{0}\left(\frac{w-K_{V}^{\prime}}{K_{V}}\right) d z\right)} \int_{-\delta}^{z} \exp \left(\int_{-\delta}^{z} \frac{w-K_{V}^{\prime}}{K_{V}} d z\right) d z+T_{b}
$$

in which, the prime means derivative in $\mathrm{z}$ direction. To simplify the relationship, we can 
varify that the w can be regarded as a constant at the base of the mixed layer (Figure (510)), $w=$ constant, and $K_{V}^{\prime} \ll w$ (Figure (5-11)). The temperature at the base of the mixed layer then becomes:

$$
\begin{aligned}
T(0) & =\frac{Q_{0}}{e^{w \delta / K_{V}}} \int_{-\delta}^{0} e^{w(z+\delta) / K_{V}} d z+T_{b} \\
& =\left.Q_{0} \frac{K_{V}}{w} e^{w z}\right|_{-\delta} ^{0}+T_{b} \\
& =Q_{0} \frac{K_{V}}{w}\left(1-e^{-\delta w}\right)+T_{b} \\
& =Q_{0} \frac{K_{V}}{w}+T_{b}
\end{aligned}
$$

in which, we negelect the small term $e^{-\delta w}$. The SST obviously changes if the subsurface temperature changes. Furthermore, the surface temperature will decrease when the vertical velocity increases. What happens in IH and IL is consistent with this relationship showing that the vertical velocity in the eastern-most grid box increases switching from IL to IH, the SST decreases correspondingly.

Alternatively, we can also consider the transient process to better understand the mechanism. Suppose we start with IL case, and the boundary condition is that $T=T_{0}$ at the surface and $T=T_{b}$ at $z=-\delta$, then the equations are:

$$
\begin{aligned}
w T_{z} & =K_{V} T_{z z} ; \\
T & =T_{0}, z=0 ; \\
T & =T_{b}, z=-\delta ; \\
V_{u} & =\frac{\tau_{y}}{\rho_{0} f} ; \\
w & =\frac{V_{u}}{\rho_{0} \triangle x}=\frac{\tau_{y}}{\rho_{0}^{2} f \triangle x}
\end{aligned}
$$

where $V_{u}$ is the offshore Ekman transport carried in the mixed layer, $T_{0}$ is the mixed layer temperature, $\tau_{y}$ is the surface wind stress (only alongshore component), and $\rho_{0}$ is the back- 
ground density. Here we consider w and $K_{V}$ are constant from the start.
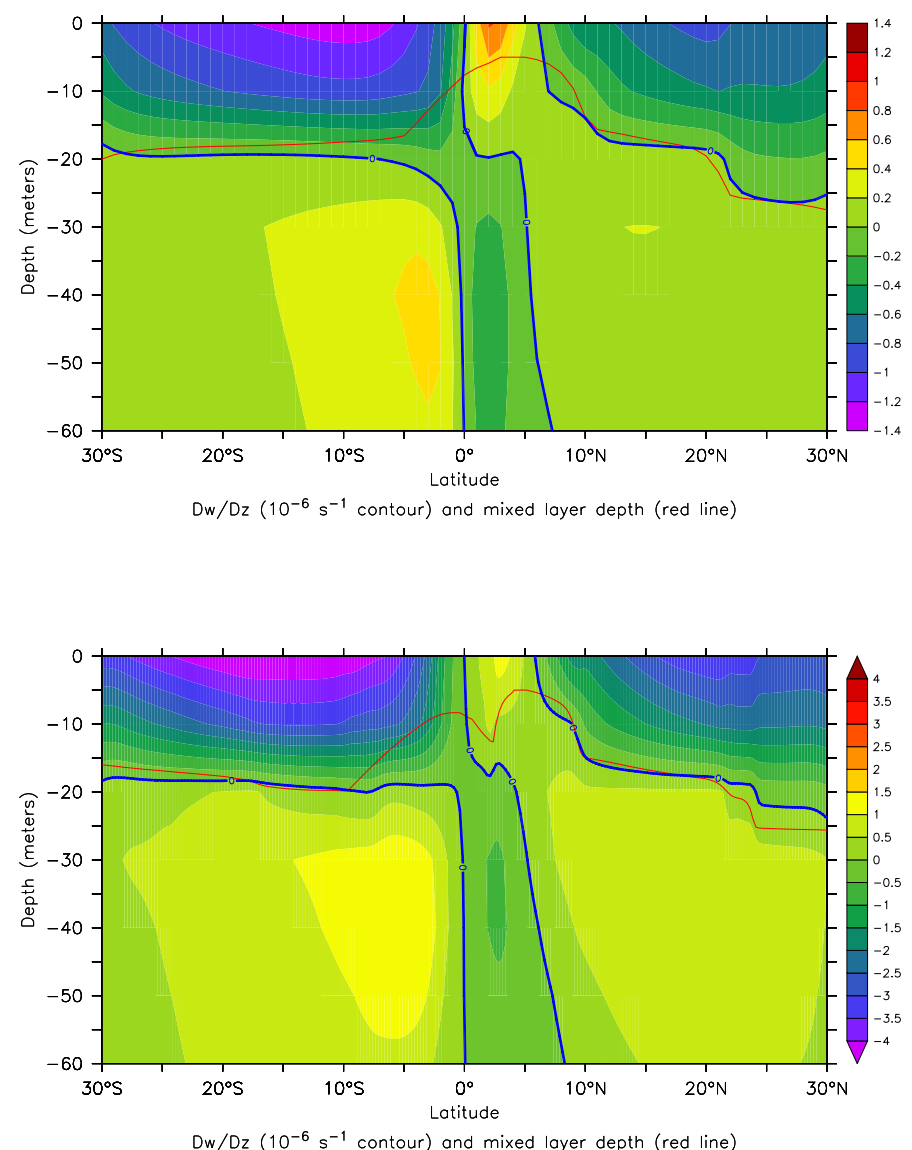

Figure 5-10: The vertical gradient of the vertical velocity (contour), and the mixed layer depth (red line) for IH (lower panel) and IL (upper panel). In the vicinity of the base of the mixed layer, $\partial w / \partial z$ is close to zero, which means we can take the vertical velocity as a local constant.

Solving equation (5.9) gives the temperature

$$
T=\left(T_{0}-T_{b}\right) \exp \left(\frac{w}{K_{V}} z\right)+T_{b}
$$

so that the vertical advection evaluated at the base of the mixed layer becomes

$$
w T_{z}=\left.\left(T_{0}-T_{b}\right) w\left(\frac{w}{K_{V}}\right) \exp \left(\frac{w}{K_{V}} z\right)\right|_{z=0}=\left(T_{0}-T_{b}\right)\left(\frac{w^{2}}{K_{V}}\right)
$$




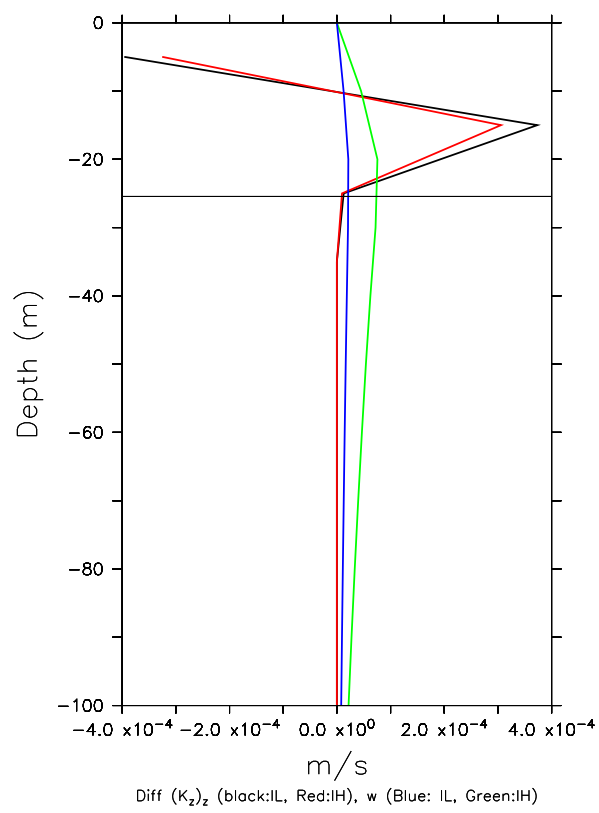

Figure 5-11: The vertical velocity (blue line for IL and green line for IH) and the vertical derivative of the diffusion coefficients (black line for IL and red line for IH) in the sidewall boundary layer at $10^{\circ} \mathrm{S}$. The black horizontal line indicates the depth of the base of the mixed layer.

in which the term on RHS represents the quadratic relationship between the vertical velocity and the vertical heat advection. The vertical velocity can be related to the surface wind stress as shown in Equation (5.9e), so that the vertical heat advection can be written as

$$
w T_{z}=\left(T_{0}-T_{b}\right)\left(\frac{\tau_{y}^{2}}{\rho_{0}^{4} f^{2} \triangle x^{2} K_{V}}\right) .
$$

The heat advection from below to the mixed layer is

$$
\begin{aligned}
Q & =\int_{y_{1}}^{y_{2}} d y C_{p} \rho_{0}\left(-w T_{z}\right) \triangle x \\
& =\frac{C}{\triangle x}, C=-\frac{C_{p}\left(T_{0}-T_{b}\right)\left(y_{2}-y_{1}\right) \tau_{y}^{2}}{K_{V} \rho_{0}^{3} f^{2}}
\end{aligned}
$$

It is obvious that the heat advection through the base of the mixed layer is proportional to 
the inverse of the zonal resolution. More cooling is carried in the smaller grid cell for the finer resolution than in the large grid cell for the coarse resolution. If the zonal resolution is suddenly increased, say to $\frac{1}{4} \triangle x$, from the previous steady state with the zonal resolution $\triangle x$, the equilibrium will be broken as the vertical advection increases and cools down the SST. As the diffusion coefficients are basically unchanged (figure not shown), the only way to reach equilibrium for the new resolution is that $T_{z}$ decreases faster than $T_{z z}$, which is true in the current study. The underlying physics is that the relationship between the vertical heat advection (entrainment) and the vertical velocity is nonlinear. The increase of the vertical velocity also raises the stratification if we keep other parameters unchanged.

This simplified formulation is a proof-of-concept. The same argument can be applied to the case with resolved boundary layers where horizontal diffusion dominates in the heat equation. We can see that $\mathrm{IHH}_{2}$ has a wider boundary layer than $\mathrm{IHH}_{1}$ (Figure 5-2). As a result, the vertical velocity is stronger in $\mathrm{IHH}_{1}$, and the SST is cooler according to (5.8), which is confirmed by the Figure (5-4).

The above discussion is based on the eastern-most grid cell adjacent to the coast. However, the different heat advection over that grid can affect the area far offshore by the fast zonal advection and wave propagation. Figure(5-12) shows that the zonal advection near the coast is significantly larger in IH than in IL. As the zonal transport is proportional to the inverse of the Coriolis parameter, $V_{u}=U H=\tau_{y} / \rho_{0} f$, the boundary influence will spread offshore over lower latitude regions which confirms the pattern of SST differences between $\mathrm{CH}$ and $\mathrm{CL}$.

\subsection{Model parameterization for the coastal boundary layer}

Parameterization is used when the model has not enough resolution to resolve the real flow. With the limited computer power, hundreds or thousands of years simulations using sophisticated climate models will be still not affordable in the near future. Modelers used the 


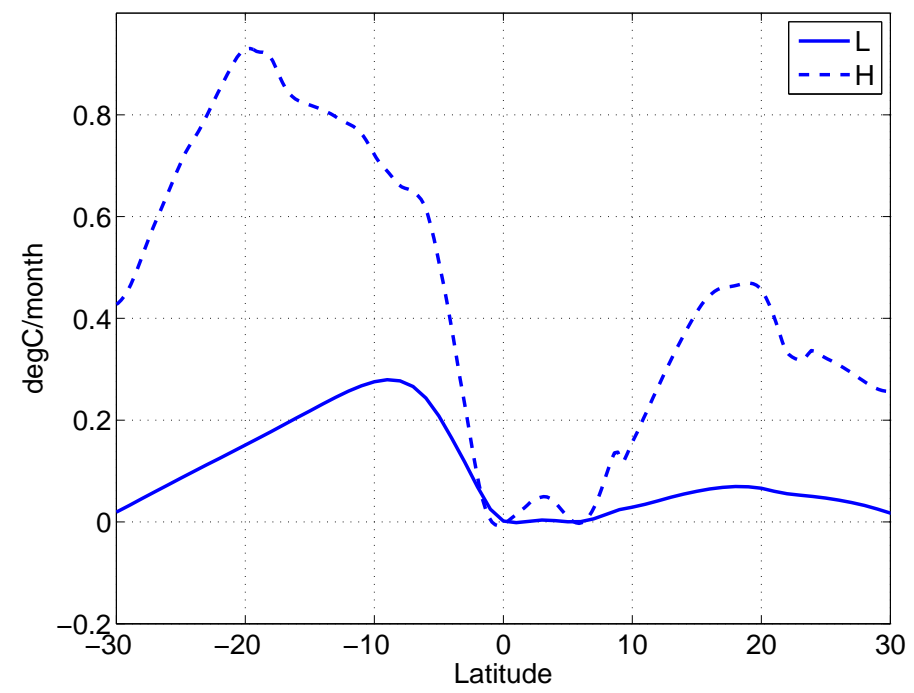

Figure 5-12: The zonal advection $\left(u T_{x}\right)$ in the surface layer $(10 \mathrm{~m})$ along the edge grid, in which the positive value represents cooling of SST. In the subtropical region, the cooling due to zonal advection is much higher in IH than in IL.

coarsely resolved ocean component in coupled models because the ocean was regarded as a responder to the atmosphere that does not need to be resolved. Recently, more and more studies have shown the importance of ocean dynamics in determining the climate variability. In this study, I also show the importance of the dynamics in the coastal boundary layer to simulate the coastal SST, which is a key factor in the Atlantic climate variability. This importance indicates that the future coupled models must either resolve or parameterize the coastal dynamics. Given the limited computer power, parameterizations may be the way to solve the problem. However, how to improve the cooling induced by the coastal upwelling within an unresolved boundary layer is still an open question.

The relationship shown by equation (5.14) indicates that the heat transport can be modified by changing values of the eddy parameterizations. Notice from equation (5.14) that the vertical heat transport is related to the inverse of the vertical diffusion coefficient, therefore it is possible to keep the heat transport to be the same for different horizontal resolutions by adjusting the vertical diffusion coefficient, that is, matching $K_{V} \triangle x$ to be the same. It means that when the horizontal resolution increases, the vertical diffusion coefficient 


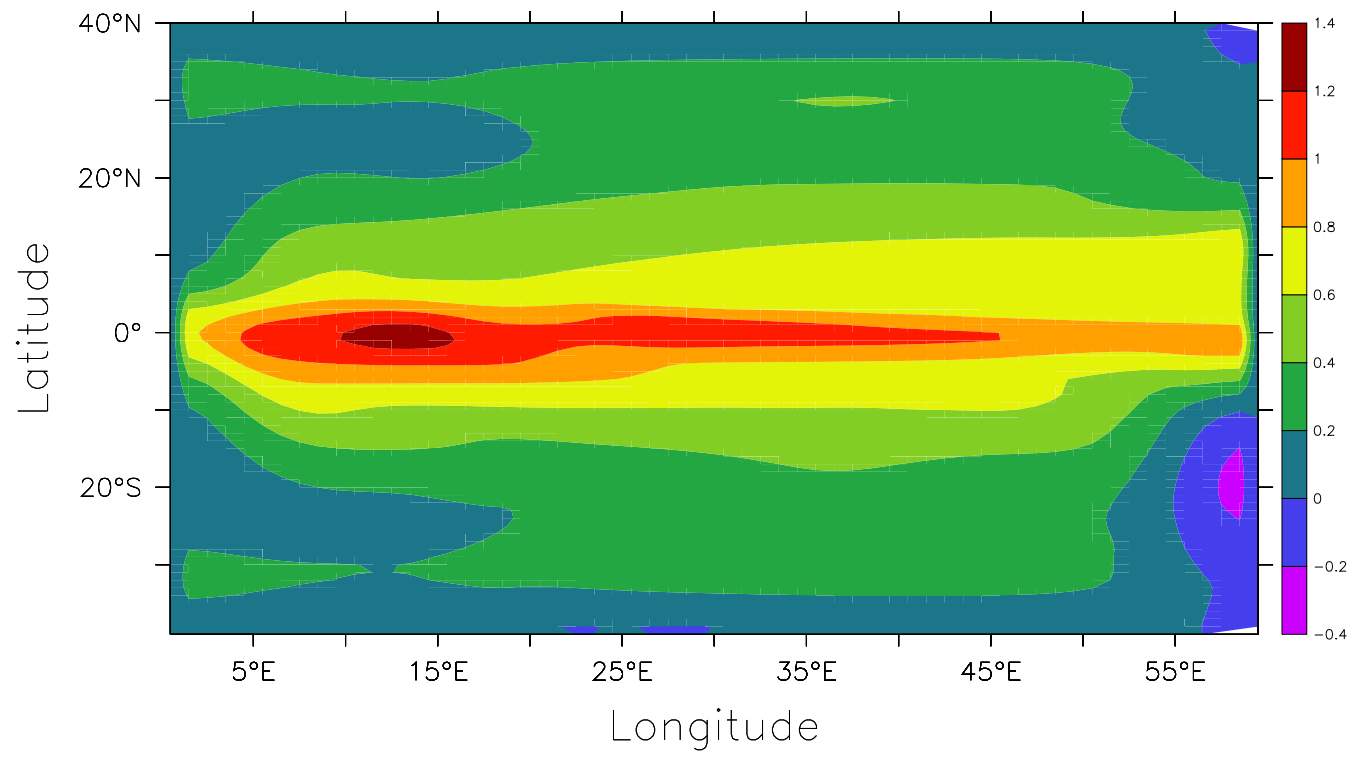

Figure 5-13: The SST difference between two simulations with everything the same but the vertical diffusion. The negative value means that reducing the vertical diffusion also cools the coastal SST over the region where the surface wind forcing is strong.

should decreases. Alternatively, the vertical advection can be increased by reducing the vertical diffusion coefficient. Figure (5-13) shows that reducing the vertical diffusion indeed cools down the coastal SST where the surface wind forcing is strong. Most of the cooler SST is over the strongest wind forcing region because the vertical heat transport is related to the quadratic term of the alongshore wind amplitude. The cooled SST produced by reducing the vertical diffusion coefficient shows that it is a feasible way to improve the coastal SST simulation by modifying the eddy parameterization based on the discussion in this study. 


\section{Chapter 6}

\section{Summary and conclusions}

Coupled models do not accurately reproduce the SST over the SWA coastal region, which generally exhibits warm biases. In this study, I first compared two simulations produced by a regional coupled model to show that increasing the horizontal resolution can reduce this well-known bias. The coastal side-wall boundary layer is responsible for the significant discrepancy between the two simulations with different horizontal resolution in the ocean model. Further investigations of coastal dynamics using a forced ocean model confirm that the warm bias is largely induced by the model deficiency in resolving the coastal side-wall boundary layer. A simple mechanism is proposed to explain the improvement of the SST simulation over the SWA coastal region.

The effect of the better-resolved ocean dynamics on the SST simulation is investigated by comparing the results generated by coupled models with varying horizontal resolutions in the ocean component. Using a regional coupled model, Seo et al. (2006) did two simulations with two different horizontal resolutions: $1^{\circ} \times 1^{\circ}$ and $0.25^{\circ} \times 0.25^{\circ}$. The model outputs are analyzed in this study, and the results of the mean state show that both simulations are capable of capturing the coastal along-shore and cross-shore currents with a vertical structure showing the first and the second baroclinic mode. The significant discrepancies occur in the vertical velocity field over the coastal side-wall boundary layer, and the hypothesis 
is proposed that the misrepresented ocean dynamics produces the underestimated vertical heat transport along the eastern boundary, and thus generating warm biases.

According to the theory about coastal boundary layer, there are mainly three coastal sidewall boundary layers which are dynamically significant. During spin-up, the flow can be regarded as inviscid and the side-wall boundary layer width is $L_{d}$, the first baroclinic Rossby radius of deformation. After the flow has reached steady state, the boundary layer width becomes $L_{d H}$ when the horizontal diffusion dominates in the heat equation, or $L_{d V}$ when the vertical diffusion dominates. The results of $\mathrm{IH} \mathrm{H}_{1,2}$, which resolve the side-wall boundary layer, show that in this simulation the horizontal diffusion dominates in the heat equation, and the boundary layer has width $L_{d H}$.

However, in simulations with a coarse horizontal resolution, the horizontal grid size is much larger than the coastal boundary layer width, which leads to the misrepresentation of processes within the boundary layer, and to the underestimated cooling. In order to avoid the grid noise, I ran two simulations using an idealized ocean model forced by a steady wind with a square domain. The results confirm what was found in the coupled model simulations that the significant discrepancy occurs within the coastal side-wall boundary layer. The simplification of the heat equation based on the analysis of model outputs leads to a simple relationship between the vertical heat transport and the zonal grid size within the coastal side-wall boundary layer. This relationship shows that without resolving the side-wall boundary layer, the upwelling-induced cooling along the coast is, to the leading order, proportional to the inverse of the zonal grid size. The consequence is that the relationship between the vertical heat transport and the upwelling velocity is nonlinear. The changes of the vertical velocity will also alter the stratification. This simple relationship explains why increasing horizontal resolution can improve the coastal SST simulation as being demonstrated in both coupled (CH and CL) and uncoupled (IH and IL) models.

Another mechanism, related to the coastal Kelvin wave dynamics, is hypothesized but not tested in the current study. Adamec and Obrien (1978) used a linear model on the equatorial $\beta$ plane to show that the local wind is insufficient to produce the upwelling in the Gulf of 
Guinea. The effects of Kelvin waves can be amplified by nonlinearities. Figure (3-6) shows that the first Kelvin baroclinic mode of the vertical velocity has a strong surface upwelling coupled with a downwelling in the subsurface layer. It is known that the transient phase of the coastal upwelling is established by coastal Kelvin waves. In steady state with a simple alongshore wind, we might however not see the effect of the coastal waves. In the fully coupled models, like $\mathrm{CH}$ and $\mathrm{CL}$, coastal waves play important roles in adjusting the upwelling. Figure (6-1) shows the Hovmuller diagrams of the surface height along the coast with a wave structure for $\mathrm{CH}$ and $\mathrm{CL}$. A rough calculation of the phase speed, $2.6 \mathrm{~m} / \mathrm{s}$, agrees with the first baroclinic Kelvin wave speed given in Philander et al. (1996) $2.4 \mathrm{~m} / \mathrm{s}$. It suggests that the remote forcing could affect the subtropical coastal region too. How these signals vary with the horizontal resolution and affect the coastal upwelling is not fully clear and it will be investigated in future work.

In this study, only steady linear solutions are considered. The coastal upwelling dynamics is simplified to be a two dimensional system. It is the zero order estimation. The system consists of surface upwelling and subsurface downwelling, surface equatorward and subsurface poleward jets. It is unknown how three dimensional currents affect the coastal upwelling in the SWA coastal region. McCreary et al. (2002) propose that the SEUC feeds the coastal upwelling, but the role of EUC, and SEUC in affecting the coastal upwelling is unclear. Additionally, the eddy activities are suppressed by model configurations including the steady wind, high viscosities and straight coastlines, but these features are ubiquitous along real eastern oceanic boundaries and play important roles in the surface heat budget (Capet et al. 2008). The question regarding what is happening in the SWA coastal region in the real ocean is still an open question, and it will also be a part of the future work. 

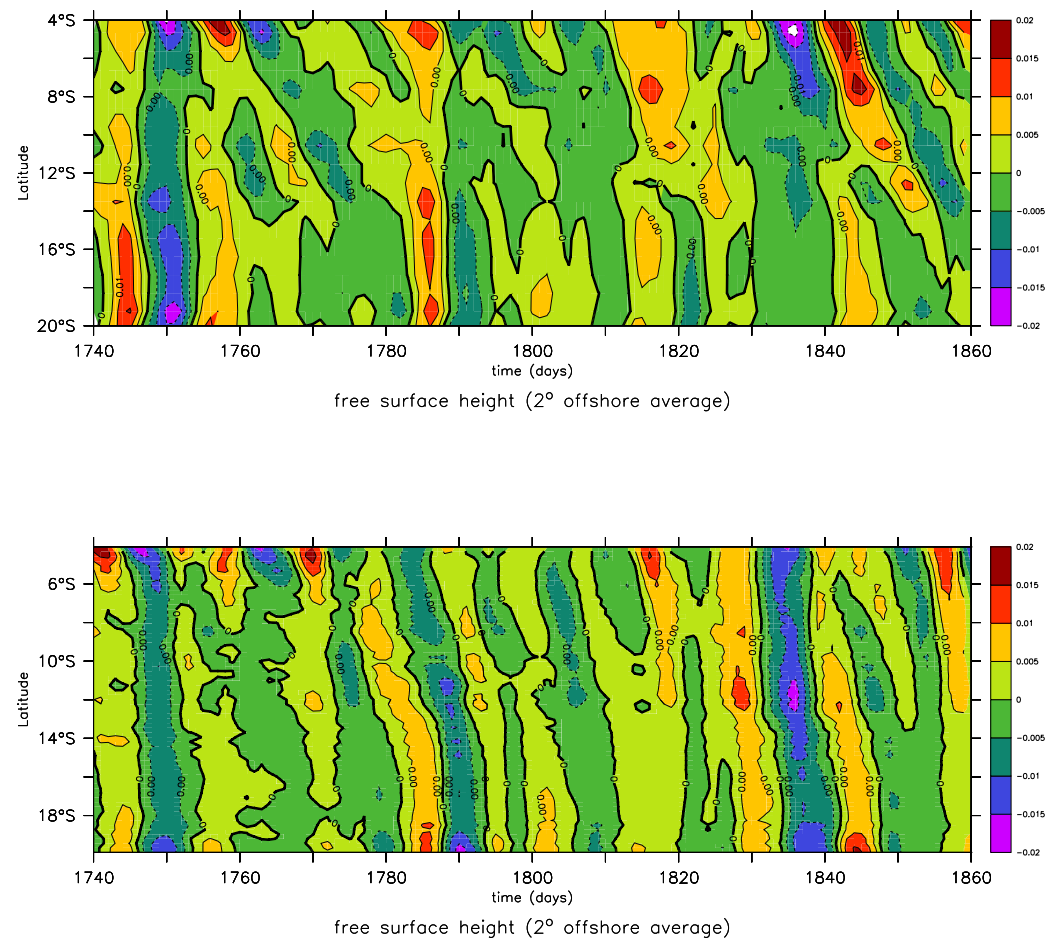

Figure 6-1: The surface height along the coasts in CL (upper) and $\mathrm{CH}$ (lower) panel. Rough calculation of the phase speed gives a value of $2.6 \mathrm{~m} / \mathrm{s}$ in $\mathrm{CH}$ which agrees quite well with the value of $2.4 \mathrm{~m} / \mathrm{s}$ in Philander et al. (1996) for the first baroclinic Kelvin mode. 


\section{Bibliography}

Adamec, D. and J. J. Obrien, 1978: Seasonal upwelling in gulf of guinea due to remote forcing. Journal of Physical Oceanography, 8, 1050-1060.

Alexander, M. A., J. D. Scott, and C. Deser, 2000: Processes that influence sea surface temperature and ocean mixed layer depth variability in a coupled model. Journal of Geophysical Research-Oceans, 105, 16823-16842.

Allen, J., 1973: Upwelling and coastal jets in a continuously stratified ocean. Journal of Physical Oceanography, 3, 245-257.

Barcilon, V. and J. Pedlosky, 1967: A unified linear theory of homogeneous and stratified rotating fluids. Journal of Fluid Mechanics, 29, 609-621.

Bjerknes, J., 1966: A possible response of the atmospheric hadley circulation to equatorial anomalies of ocean temperature. Tellus, 18, 820-829.

Bryan, F., 1987: Parameter sensitivity of primitive equation ocean general-circulation models. Journal of Physical Oceanography, 17, 970-985.

Capet, X., J. McWilliams, M. Molemaker, and A. Shchepetkin, 2008: Mesoscale to Submesoscale Transition in the California Current System. Part I: Flow Structure, Eddy Flux, and Observational Tests. Journal of Physical Oceanography, 38, $29-43$.

Carton, J., X. Cao, B. Giese, and A. da Silva, 1996: Decadal and interannual sst variability in the tropical atlantic ocean. Journal of Physical Oceanography, 26, 1165-1175.

Chang, P., R. Saravanan, and L. Ji, 2003: Tropical atlantic seasonal predictability: The roles of el nino remote influence and thermodynamic air-sea feedback. Geophysical Research Letters, 30(10), 1501, doi:10.1029/2002GL016119.

Chang, P., R. Saravanan, L. Ji, and G. Hegerl, 2000: The effect of local sea surface temperatures on atmospheric circulation over the tropical atlantic sector. Journal of Climate, 13, 2195-2216. 
Chiang, J. C. H., Y. Kushnir, and A. Giannini, 2002: Deconstructing atlantic intertropical convergence zone variability: Influence of the local cross-equatorial sea surface temperature gradient and remote forcing from the eastern equatorial pacific. Journal of Geophysical Research-Atmospheres, 107, 4004, doi:10.1029/2000JD000307.

Conkright, M. E., H. E. Garcia, T. D. O’Brien, R. A. Locarnini, T. P. Boyer, C. Stephens, and J. Antonov, 2002: World Ocean Atlas 2001. U.S. Government Printing Office.

da Silva, A., C. Young, and S. Levitus, 1994: Atlas of surface marine data 1994 noaa atlas nesdis. United States National Environmental Satellite Data and Information Service. NOAA atlas NESDIS ; v. 1. Algorithms and procedures - v. 2. Anomalies of directly observed quantities - v. 3. Anomalies of heat and momentum fluxes - v. 4. Anomalies of fresh water fluxes - v. 5. Anomalies of miscellaneousderived quantities.

Danabasoglu, G., W. G. Large, J. J. Tribbia, P. R. Gent, B. P. Briegleb, and J. C. McWilliams, 2006: Diurnal coupling in the tropical oceans of ccsm3. Journal of Climate, 19, 2347-2365.

Davey, M. K., M. Huddleston, K. R. Sperber, P. Braconnot, F. Bryan, D. Chen, R. A. Colman, C. Cooper, U. Cubasch, P. Delecluse, D. DeWitt, L. Fairhead, G. Flato, C. Gordon, T. Hogan, M. Ji, M. Kimoto, A. Kitoh, T. R. Knutson, M. Latif, H. Le Treut, T. Li, S. Manabe, C. R. Mechoso, G. A. Meehl, S. B. Power, E. Roeckner, L. Terray, A. Vintzileos, R. Voss, B. Wang, W. M. Washington, I. Yoshikawa, J. Y. Yu, S. Yukimoto, S. E. Zebiak, and Gn, 2002: Stoic: a study of coupled model climatology and variability in tropical ocean regions. Climate Dynamics, 18, 403-420.

Furue, R., J. McCreary Jr, Z. Yu, and D. Wang, 2007: Dynamics of the Southern Tsuchiya Jet. Journal of Physical Oceanography, 37, 531-553.

Gu, D. and S. G. H. Philander, 1997: Interdecadal climate fluctuations that depend on exchanges between the tropics and extratropics. Science, 275(5301), 805-807.

Haney, R., 1971: Surface thermal boundary condition for ocean circulation models. Journal of Physical Oceanography, 1, 241-248.

Huang, B., 2004: Remotely forced variability in the tropical atlantic ocean. Climate Dynamics, 23, 133-152.

Jochum, M. and P. Malanotte-Rizzoli, 2001: Influence of the Meridional Overturning Circulation on Tropical-Subtropical Pathways. Journal of Physical Oceanography, 31, 1313-1323.

— 2004: A New Theory for the Generation of the Equatorial Subsurface Countercurrents. Journal of Physical Oceanography, 34, 755-771. 
Jochum, M., R. Murtugudde, R. Ferrari, and P. Malanotte-Rizzoli, 2005: The Impact of Horizontal Resolution on the Tropical Heat Budget in an Atlantic Ocean Model. Journal of Climate, 18, 841-851.

Johnson, G. and M. McPhaden, 1999: Interior pycnocline flow from the subtropical to the equatorial pacific ocean. Journal of Physical Oceanography, 29, 3073-3089.

Johnson, H. and D. Marshall, 2002: A theory for the surface atlantic response to thermohaline variability. Journal of Physical Oceanography, 32, 1121-1132.

Juang, H., S.-Y. Hong, and M. Kanamitsu, 1997: The ncep regional spectral model: An update. Bulletin of the American Meteorological Society, 78, 2125-2143.

Juang, H. and M. Kanamitsu, 1994: The nmc nested regional spectral model. Monthly Weather Review, 122, 3-26.

Kanamitsu, M., W. Ebisuzaki, J. Woollen, S. K. Yang, J. J. Hnilo, M. Fiorino, G. L. Potter, and $\mathrm{Vu}, 2002$ : Ncep-doe amip-ii reanalysis (r-2). Bulletin of the American Meteorological Society, 83, 1631-1643.

Kleeman, R., J. McCreary, and B. Klinger, 1999: A mechanism for generating enso decadal variability. Geophysical Research Letters, 26, 1743-1746.

Large, W. G. and G. Danabasoglu, 2006: Attribution and impacts of upper-ocean biases in ccsm3. Journal of Climate, 19(11), 2325-2346.

Large, W. G., J. C. McWilliams, and S. C. Doney, 1994: Oceanic vertical mixing - a review and a model with a nonlocal boundary-layer parameterization. Reviews of Geophysics, 32, 363-403.

Lindzen, R. and S. Nigam, 1987: On the role of sea surface temperature gradients in forcing low level winds and conver-gence in the tropics. Journal of Atmospheric Sciences, 44, 2440-2458.

Liu, Z. and S. G. H. Philander, 1995: How different wind stress patterns affect the tropicalsubtropical circulations of the upper ocean. Journal of Physical Oceanography, 25(4), 449-462.

Marshall, J., C. Hill, L. Perelman, and A. Adcroft, 1997: Hydrostatic, quasi-hydrostatic, and nonhydrostatic ocean modeling. Journal of Geophysical Research-Oceans, 102, 5733-5752.

McCreary, J. P., P. Lu, and Z. Yu, 2002: Dynamics of the pacific subsurface countercurrents. Journal of Physical Oceanography, 32, 2379-2404. 
Mechoso, C., A. Robertson, N. Barth, M. Davey, P. Delecluse, P. Gent, S. Ineson, B. Kirtman, M. Latif, H. Treut, et al., 1995: The Seasonal Cycle over the Tropical Pacific in Coupled Ocean-Atmosphere General Circulation Models. Monthly Weather Review, 123, 2825-2838.

Pedlosky, J., 1987: Geophysical Fluid Dynamics, Springer, chapter 8. 2nd edition, 640.

Philander, S. G. H., D. Gu, D. Halpern, G. Lambert, N. C. Lau, T. Li, R. C. Pacanowski, and Wf, 1996: Why the itcz is mostly north of the equator. Journal of Climate, 9, 2958-2972.

Seo, H., M. Jochum, R. Murtugudde, A. J. Miller, and Gj, 2006: Effect of ocean mesoscale variability on the mean state of tropical atlantic climate. Geophysical Research Letters, 33, 4 .

Seo, H., A. J. Miller, and J. O. Roads, 2007: The scripps coupled ocean-atmosphere regional (scoar) model, with applications in the eastern pacific sector. Journal of Climate, 20, 381-402.

Shchepetkin, A. F. and J. McWilliams, 2005: The regional oceanic modeling system (roms): A split-explicit, free-surface, topography-following-coordinate ocean model. Ocean Modelling, 9, 347-404.

Stewartson, K., 1957: On almost rigid rotations. Journal of Fluid Mechanics, 3, 17-26.

Xie, S. and S. Philander, 1994: A coupled ocean-atmosphere model of relevance to the itcz in the eastern pacific. Tellus, 46A, 340-350.

Yeager, S., C. Shields, W. Large, and J. Hack, 2006: The Low-Resolution CCSM3. Journal of Climate, 19, 2545-2566.

Yoon, J. and S. Philander, 1982: The generation of coastal undercurrents. Journal of Oceanographical Society of Japan, 38, 215-224. 